\title{
Delay, Reliability, and Throughput Based QoS Profile: A MAC Layer Performance Optimization Mechanism for Biomedical Applications in Wireless Body Area Sensor Networks
}

\author{
Muhammad Sajjad Akbar, ${ }^{1}$ Hongnian Yu, ${ }^{1}$ and Shuang Cang ${ }^{2}$ \\ ${ }^{1}$ Faculty of Science \& Technology, Bournemouth University, Poole House, Talbot Campus, Fern Barrow, Poole BH12 5BB, UK \\ ${ }^{2}$ Faculty of Management, Bournemouth University, Poole House, Talbot Campus, Fern Barrow, Poole BH12 5BB, UK
}

Correspondence should be addressed to Hongnian Yu; yuh@bournemouth.ac.uk

Received 11 August 2015; Revised 21 October 2015; Accepted 9 November 2015

Academic Editor: Yasuko Y. Maruo

Copyright (C) 2016 Muhammad Sajjad Akbar et al. This is an open access article distributed under the Creative Commons Attribution License, which permits unrestricted use, distribution, and reproduction in any medium, provided the original work is properly cited.

\begin{abstract}
Recently, increasing demand for remote healthcare monitoring systems poses a specific set of Quality of Services (QoS) requirements to the MAC layer protocols and standards (IEEE 802.15.6, IEEE 802.15.4, etc.) of Wireless Body Area Sensor Networks (WBASNs). They mainly include time bounded services (latency), reliable data transmission, fair channel distribution, and specified data rates. The existing MAC protocols of WBASNs are lack of a specific set of QoS. To address this, the paper proposes a QoS profile named delay, reliability, and throughput (DRT). The QoS values computed through DRT profile provide maximum reliability of data transmission within an acceptable latency and data rates. The DRT is based on the carrier sense multiple access with collision avoidance (CSMA/CA) channel access mechanism and considers IEEE 802.15.4 (low-rate WPAN) and IEEE 802.15.6 (WBASN). Further, a detailed performance analysis of different frequency bands is done which are standardized for WBASNs, that is, $420 \mathrm{MHz}$, $868 \mathrm{MHz}, 2.4 \mathrm{GHz}$, and so forth. Finally, a series of experiments are conducted to produce statistical results for DRT profile with respect to delay, reliability, and packet delivery ratio (PDR). The calculated results are verified through extensive simulations in the CASTALIA 3.2 framework using the OMNET++ network simulator.
\end{abstract}

\section{Introduction}

With the increasing interest of medical applications in remote healthcare, WBASN (Wireless Body Area Sensor Network) has received significant attention from both academic researchers and industry practitioners [1-4]. Personal assistance [5], patient monitoring [6], environment, and military [7] are considered as a popular application area for WBASN. A WBASN is composed of sensor nodes, which possess the characteristics like low power, miniaturization, wearability, light weight, and so forth. For medical applications, electrocardiograph (ECG), electroencephalogram (EEG), electromyography (EMG), accelerometer, gyroscope, pulse oximeter, blood pressure, temperature, barometer, and heart rate monitoring sensor are used. These sensor nodes are robust and capable of continuous monitoring with a limited memory. These wearable sensor nodes continuously monitor the physiological data and send them to a coordinator node (Personal Digital Assistant (PDA) or any other suitable node) before memory fills for preprocessing using radio frequency (RF) signals. The coordinator node is placed on the body or near body. A Central Server (CS) is used to keep data records for different patients to provide them with emergency services and feedback. The overall WBASN architecture contains three tiers, including wearable sensors, on-body coordinator, and CS. In this three-tier architecture, communication is conducted at two stages, that is, from the sensor node to the coordinator and from the coordinator to the CS. One of the purposes of WBASN is to provide remote healthcare to decrease the hospitalization as the number of elderly people 
is increasing yearly [8]. Research work regarding design, evaluation, and implementation of WBASN is conducted in [9-13]. The wearable and implantable WBASN has two main categories for healthcare applications [14, 15]. The implantable WBASN is used for telecare and telemedicine, whereas the wearable WBASN is used for medical and nonmedical applications [16]. In remote healthcare applications, WBASN research focuses on four main areas including ultralow power processing, signal processing, communication protocols, and lightweight sensor nodes [17, 18].

WBASN is a subclass of wireless sensor networks (WSN). Various communicating protocols and mechanisms are developed for WSN but their use in WBASN is not suitable due to the different environment a human body has [19]. These different characteristics are as follows: (a) there is limited bandwidth due to the fact that the available bandwidth is short-range, changing, fading, and noisy and in a limited space; (b) sensor nodes and devices used in WBASNs are heterogeneous with respect to QoS requirements; (c) sensor nodes should work on low power because high power can be harmful for a human; (d) mobility is involved with this small network as it moves with the body part movements. Wearable health monitoring systems require defined and strict medical criteria as they work under ergonomic and hardware limitations. Mainly these constraints include data rate, delay, QoS, and power consumption. ISO/IEEE 11073 defines a few classes of medical applications with their latency and data rate requirements. For latency the acceptable limit is $250 \mathrm{~ms}$ for the most medical applications. For data rates different applications require different data rates; for example, blood pressure, blood saturation, heart rate, and temperature demand less than $10 \mathrm{kbps}$, whereas EMG, audio, and video require more than $100 \mathrm{kbps}$. Table 1(a) highlights those requirements for different medical applications. Table 1(b) describes different WBASN projects.

IEEE 802.15.4 standard defines the data link layer (DLL) and physical layer for low-rate wireless personal area networks (LR-WPANs). It is developed and maintained by the IEEE 802.15 working group, which provides the basis for wireless personal area networks (WPANs). IEEE 802.15.4 defines two types of devices, that is, full function device (FFD) and reduced function device (RFD). FFD supports full functionalities and can act as a network coordinator, whereas RFD supports limited functionality and usually is used by a network edge device as it consumes low power. The role of the network coordinator is to manage a network (usually deployed in a star topology) as a central controller sends and receives data through it. The IEEE 802.15 .4 physical layer is capable of using three frequencies bands: (a) 2.4 to $2.4835 \mathrm{GHz}$ with 16 channels; (b) 902 to $928 \mathrm{MHz}$ using 10 channels; (c) 868 to $868.6 \mathrm{MHz}$ with one channel. DLL consists of two sublayers, medium access control (MAC) and logical link control (LLC). The LLC is defined in IEEE 802.2 and is used in 802 with the same functionality. The MAC handles various activities including beacon management, guaranteed time slots (GTS) management, and channel access mechanism.

The task group for 802.15.6 was established by IEEE in November 2007 for standardization of WBASNs and was approved in 2012. This standard works in and around the human body and focuses on operating at lower frequencies and a short range. This standard focuses on designing a communication standard for MAC and the physical layer to support different applications, that is, medical and nonmedical applications. It defines a MAC layer that can operate with three different physical layers, that is, human body communication (HBC), ultra wideband (UWB), and narrow band (NB) [1].

For optimal performance WBASN applications demand a set of QoS combinations at a given time from the MAC layer. The current protocols and standards lack this aspect. Section 2 reviews the existing MAC layer protocols for healthcare. Section 3 presents the fundamental carrier sense multiple access with collision avoidance (CSMA/CA) mechanism for IEEE 802.15.4 and IEEE 802.15.6. Section 4 discusses the delay, reliability, and throughput (DRT) profile in detail with the help of numerical modeling. Section 5 provides simulations and validation results.

\section{MAC Protocols for Medical Applications and Healthcare Systems}

WBASNs [2] use two types of MAC mechanisms for periodic and urgent data traffic, that is, CSMA/CA and time-division multiple access (TDMA). CSMA/CA is the contention based channel access where nodes need to compete for the channel in the contention access period (CAP), whereas TDMA is a contentionless and scheduled (on time slots) channel access mechanism.

Table 2 shows that CSMA/CA is suitable for the networks where both urgent and periodic data need to be transmitted. Moreover, it is suitable in dynamic environments where a node can be added to network at any time.

In the last few years, various research works related to WBASNs protocols have been conducted. Figure 1 shows a MAC protocol classification for healthcare applications under the existing IEEE MAC and PHY standards. It is observed that the most used protocols work under the IEEE 802.15.4 and IEEE 802.15.6 standards and perform well under the CSMA/CA channel access mechanisms.

Performance of CSMA/CA mainly depends on four parameters, namely, minimum backoff exponent (macMinBE), maximum backoff exponent (macMaxBE), contention window (CW) value, and maximum number of backoffs (macMaxCSMAbackoffs). IEEE 802.15.4 defines a fix backoff range; that is, default value of macMinBE is 3 and macMaxBE is 5 . These values clearly describe the limit of backoff interval between $2^{3}$ and $2^{5}$. Changes of these values will affect the network performance [20]. Decreasing the values for macMinBE and macMaxBE will reduce the waiting time of a node in the case where clear channel assessment (CCA) detects the busy channel. Ultimately, when a node tries the channel access with less waiting time, there is a chance that the node's throughput gets the increase. However, these decrements can also reduce the network performance. If there are different nodes that are trying to access the channel, the collision probability will increase as 
TABLE 1: (a) Requirements of medical applications [1-3]. (b) Projects under WBASNs.

(a)

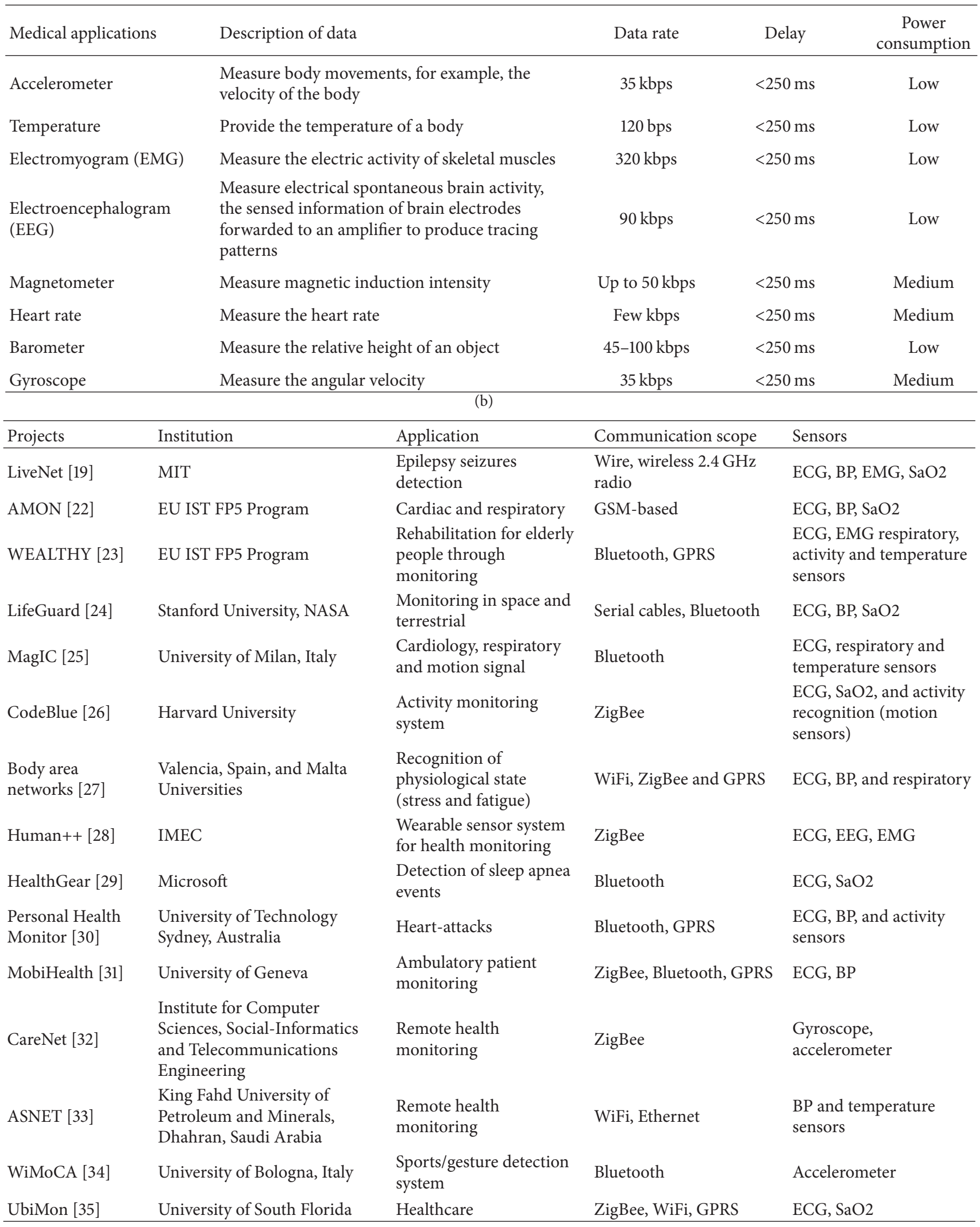


TABLE 2: Comparison of CSMA/CA and TDMA.

\begin{tabular}{lcc}
\hline Comparative parameters & CSMA/CA & TDMA \\
\hline Synchronization & Not essential & Essential \\
Packet failure & Cause less delay & Cause more delay \\
Dynamic & Work well to handle network changes & Perform poorly in a dynamic environment \\
Bandwidth utilization & Medium & High \\
Power consumption & Medium & Low-medium \\
\hline
\end{tabular}

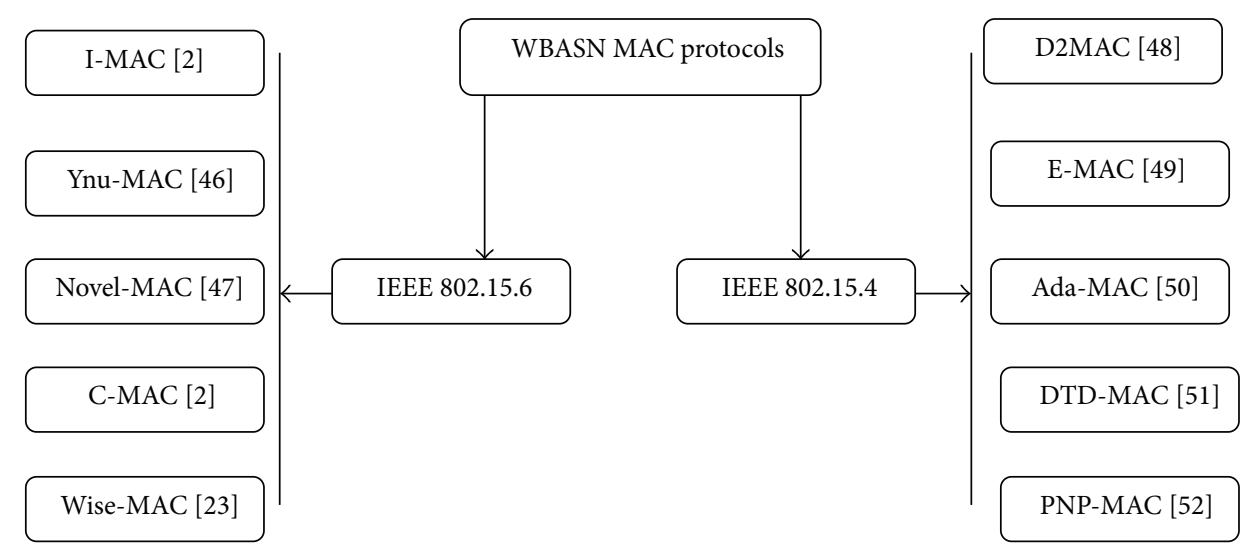

FIgURE 1: IEEE 802.15.4 and IEEE 802.15.6 based WBASN MAC protocols [2, 23, 46-52].

every node is trying to access the channel in a short interval. Overall, all nodes will have delay and less throughput. On the other hand, if the values of $\mathrm{BE}$ are increased, there is more chance of the delay and less network throughput. However, higher values of BE decrease the collision probabilities and reduce the retransmission as well.

The contention window is another important parameter for slotted CSMA/CA, which creates the waiting time after the successful CCA. The standard recommends that this delay should be equal to two times of CCA attempts. The purpose of this wait is to reduce the collision probability, by protecting the acknowledgement frame of the receiver. Usually, the receiver sends the acknowledgement after the defined interval which is between 12 and 31 symbols $(20$ symbols = one backoff period). So the time for one CCA can cause a collision within a new transmission (newly transmitted packet and its acknowledgement). The higher values of $\mathrm{CW}$ reduce the chances of collision but can cause delays. However, if the device does not need an acknowledgement, then the value of $\mathrm{CW}$ can be chosen flexibly to reduce delays.

$\mathrm{NB}$ represents the number of CSMA/CA backoff attempts and is considered as one of the important channel access parameters. The default value is 4 which means after 4 times if the device under the CSMA/CA does not get the channel access the data packet will be dropped. The parameter helps to increase the packet delivery ratio.

Table 3 describes the different optimization mechanisms by manipulating these limitations of fix values of MAC parameters and applied to existing WBASNs protocols and IEEE standards, that is, IEEE 802.15.4 and IEEE 802.15.6.

The higher the number of retransmissions is, the better the reliability of the data transmission is. Retransmission occurs when a sending device does not receive acknowledgement from the receiver. There are different reasons for not receiving the acknowledgement, that is, data packet loss due to collision on receiving side, acknowledgement lost, late reception of acknowledgement, and so forth. The high number of retransmissions ensures the reliability, but at the same time it causes delay in network performance as retransmissions involve the channel access to the same packet and also it requires the bandwidth which will affect the performance of other nodes. The traditional profiles of IEEE 802.15.4 and IEEE 802.15.6 impose restrictions by giving default values for these MAC layer parameters. Table 4 gives the default profile values.

Table 3 shows that IEEE 802.15.4 and IEEE 802.15.6 standards are capable of optimized performance in terms of delay, packet delivery, and reliability of WBASN applications by adjusting the different MAC parameter values (BE, CW, superframe order (SO), and retransmissions). Most of the optimization mechanisms applied in Table 4 usually consider one of the QoS aspects of the MAC layer, whereas a WBASN demands a set of QoS parameters in a given time. In this context, research develops a DRT profile by considering different frequency bands and data rates of IEEE 802.15.4 and IEEE 802.15.6. This profile helps to increase the performance of these standards. 
TABLE 3: Existing optimization mechanisms of the MAC layer CSMA/CA WBASN.

\begin{tabular}{|c|c|c|}
\hline Paper & $\begin{array}{c}\text { Considered MAC } \\
\text { parameters }\end{array}$ & Contribution \\
\hline $\begin{array}{l}\text { Adaptive and real time } \\
\text { protocols [20] }\end{array}$ & Backoff exponent (BE) & $\begin{array}{l}\text { Analysis of macMinBE and macMaxBE values: by increasing these } \\
\text { values the delay increases and the throughput decreases; however } \\
\text { collision probability decreases and vice versa }\end{array}$ \\
\hline Analysis of CAP [36] & $\mathrm{BE}$ & $\begin{array}{l}\text { For large networks, the throughput is independent of the BE initial } \\
\text { value; however, for small networks it generates high impact }\end{array}$ \\
\hline $\begin{array}{l}\text { A comprehensive analysis } \\
\text { of the MAC unreliability } \\
\text { problem [37] }\end{array}$ & Contention windows (CW) & $\begin{array}{l}\text { Propose CW values for time critical applications; higher values of CW } \\
\text { reduce collision chances; however, for time critical applications where } \\
\text { ACK is disabled the CW is set to lower values for fast communications }\end{array}$ \\
\hline $\begin{array}{l}\text { MAC unreliability problem } \\
\text { in IEEE 802.15.4 WSN [38] }\end{array}$ & Retransmissions & $\begin{array}{l}\text { In ideal channel conditions, when the number of retransmissions is } \\
\text { increased from } 1 \text { to } 2 \text {, a significant improvement is observed in packet } \\
\text { delivery; however, there is no significant improvement in packet } \\
\text { delivery if the number of retransmissions is increased from } 2 \text { to } 7\end{array}$ \\
\hline $\begin{array}{l}\text { Performance evaluation } \\
\text { with different backoff } \\
\text { ranges [39] }\end{array}$ & $\mathrm{BE}$ & $\begin{array}{l}\text { Provide priority-based QoS by assigning different BE values to } \\
\text { different applications }\end{array}$ \\
\hline $\begin{array}{l}\text { Priority-based delay } \\
\text { mitigation for event } \\
\text { monitoring [40] }\end{array}$ & $\begin{array}{l}\text { MAC frame modification, } \\
\text { CCA }\end{array}$ & $\begin{array}{l}\text { Collision avoidance-based priority mechanism by using one time } \\
\text { CCA }\end{array}$ \\
\hline $\begin{array}{l}\text { Priority-based service } \\
\text { differentiation scheme [41] }\end{array}$ & CW & Contention Windows Differentiation (CWD) mechanism is proposed \\
\hline $\begin{array}{l}\text { MAC protocol } \\
\text { implementation [42] }\end{array}$ & Superframe order (SO) & $\begin{array}{l}\text { Conduct a study by applying different values of superframe order } \\
\text { (SO); results show that high values of SO provide high throughput, } \\
\text { whereas low SO values result in less delay }\end{array}$ \\
\hline $\begin{array}{l}\text { Performance evaluation } \\
\text { with backoff }[43,44]\end{array}$ & $\mathrm{BE}$ & $\begin{array}{l}\text { Propose an Adaptive Backoff Exponent (ABE) mechanism which } \\
\text { reduces the devices probability to reduce collisions }\end{array}$ \\
\hline $\begin{array}{l}\text { Comprehensive simulation } \\
\text { study for CSMA/CA [45] }\end{array}$ & $\mathrm{BE}$ & $\begin{array}{l}\text { Higher values of macMinBE and macMaxBE lead towards higher } \\
\text { packet delivery ratios; however, the delay will be increased as well }\end{array}$ \\
\hline
\end{tabular}

TABle 4: Default MAC parameters setting [1].

\begin{tabular}{lc}
\hline Parameters & Default values \\
\hline macMinBE & 3 \\
macMaxBE & 5 \\
macMaxCSMABackoff & 4 \\
macMaxFrameRetries & 2 \\
\hline
\end{tabular}

\section{Investigation of IEEE 802.15.4 and IEEE 802.15.6}

3.1. IEEE 802.15.4. Figure 2 shows the frame structure which mainly contains a MAC protocol data unit (MPDU) and a physical service data unit (PSDU). The PSDU consists of four fields: (a) preamble (32-bit length for symbol synchronization); (b) packet delimiter (8 bits long) for frame synchronization; (c) physical header (8 bits, defining the PSDU length); (d) PSDU containing the payload of length 0 to 127 bytes. The MPDU carries three fields, that is, a threebyte MAC header (containing different information fields like frame control field (FCF), duration, sequence control information, and address field), frame body of variable length, and MAC footer (MFR).

Overall, the standard defines four types of frame, namely, beacon frame (mostly the coordinator is used for beacon transmission), data frame (used for transmission of data), acknowledgement frame, and MAC command frame (for all MAC peer entity control transfers).

The nonbeacon (beaconless) mode and beacon mode are two basic transmission modes of IEEE 802.15.4 [1, 2]. In a nonbeacon mode, when a node wants to transmit data, it will sense the channel (unslotted CSMA/CA) and if the channel is free then it will transmit data and receive acknowledgement (acknowledgement is optional). The coordinator is set in the waiting state in this mode. If the channel is busy then the device needs to wait for a random amount of time defined in the standard. This mode is suitable for those sensors which usually like to sleep for most of the time. They only send data on happening of an event. There is no need of any synchronization process from the coordinator; the coordinator is only responsible for the association and disassociation. Although this mode provides scalability, long battery life, and self-organization, it does not provide any guarantees for data transmissions.

Figure 3 shows the data flow diagram for the beaconless mode. The nonbeacon based CSMA/CA is mainly based on three variables, namely, backoff exponent (BE), backoff delays, and number of backoff (NB) attempts. BE is used for backoff delay calculation. The backoff period can be defined as the time required for sending 20 symbols (each symbol represents 4 bits). The number of backoff periods that a device 


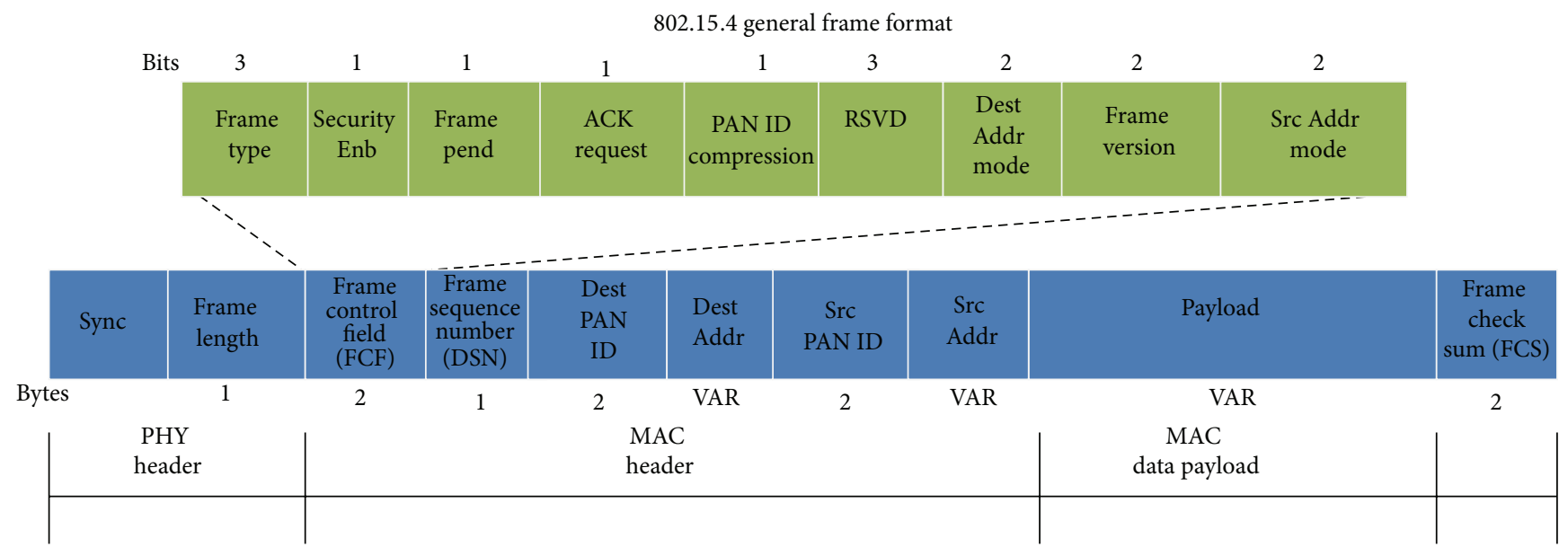

FIGURE 2: Frame structure of IEEE 802.15.4.

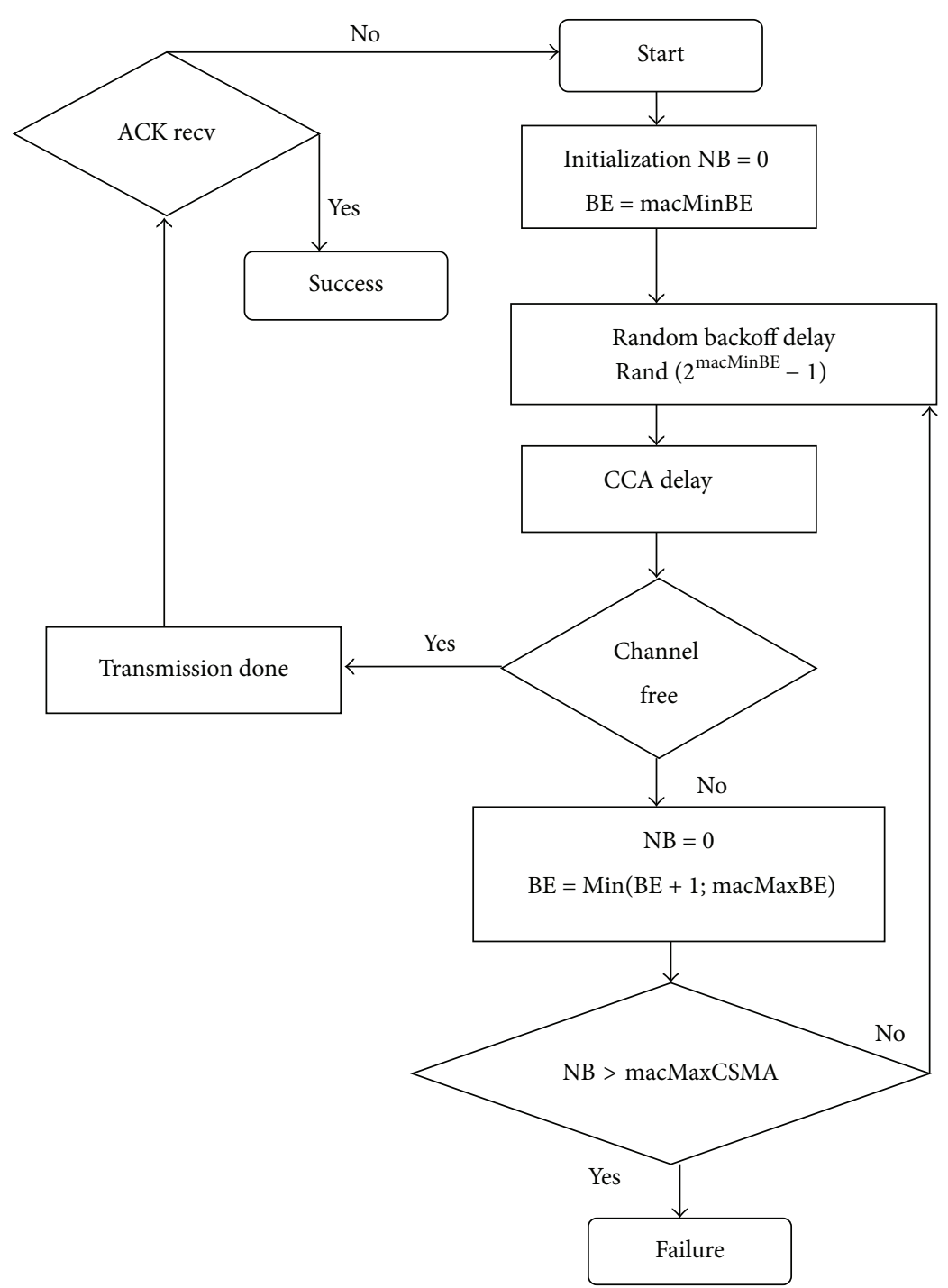

FIGURE 3: Data transmission flow chart of beaconless mode of IEEE 802.15.4. 


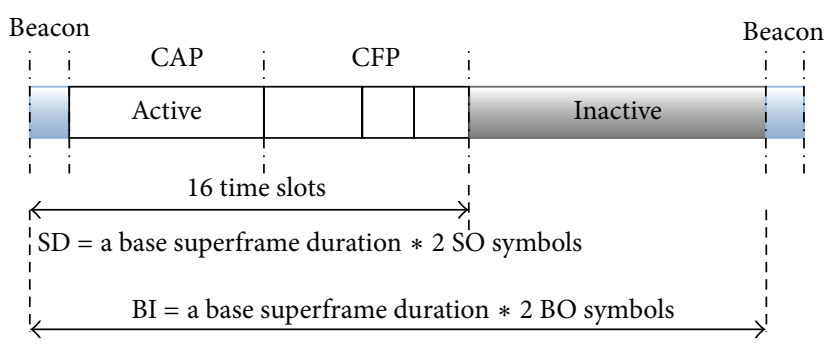

FIgURE 4: Beacon frame format of IEEE 802.15.4 [2, 3].

waits depends on the random value that falls between 0 and $2^{\mathrm{BE}}-1$, where the value of $\mathrm{BE}$ is initialized with a variable of the CSMA/CA algorithm macMinBE. NB represents the number of backoffs attempts while accessing the channel. Figure 3 shows the data flow diagram for the beaconless mode.

In the beacon mode, the coordinator sends a beacon periodically to the devices for synchronization. By receiving the beacons, the devices get knowledge of two important events, that is, superframe duration (coordinator's activity period) and when the device can do data transmission.

Figure 4 shows the superframe structure. The superframe is delimited by using beacon frames and Beacon Interval (BI) is used to define the time between two consecutive beacons. The MAC attributes beacon order (BO) and SO represent the $\mathrm{BI}$ length and superframe duration (SD). The superframe is divided into two portions, namely, active portion and inactive portion (optionally).

The active portion consists of 16 equally spaced slots, known as superframe time slots. For the active portion, the duration of 960 symbols (one symbol $=4$ bits) is fixed by IEEE 802.15 .4 , which corresponds to $15.36 \mathrm{~ms}$ (with the assumption of $250 \mathrm{kbps}$ in $2.4 \mathrm{GHz}$ band). The active portion of the superframe is divided into two parts: CAP (contention access period) and CFP (contention-free period). According to IEEE 802.15.4 the BI defines the length of the superframe as follows:

$$
\begin{aligned}
& \mathrm{BI}=\mathrm{a} \text { base superframe duration } * 2 \mathrm{BO} \text { symbols } \\
& \qquad \text { for } 0 \leq \mathrm{BO} \leq 14 .
\end{aligned}
$$

The active period follows the following formula:

$$
\begin{aligned}
& \mathrm{SD}=\text { a base superframe duration } * 2 \mathrm{SO} \text { symbols } \\
& \qquad \text { for } 0 \leq \mathrm{SO} \leq \mathrm{BO} \leq 14 .
\end{aligned}
$$

CAP is similar to the nonbeacon mode in access except it is bound to access the channel within the time slots (transmission can start at the start of time slot not in the middle).

Figure 5 describes the flow chart for the slotted mode. The slotted CSMA/CA algorithm mostly depends on three variables, namely, $\mathrm{BE}, \mathrm{CW}$, and $\mathrm{NB}$. $\mathrm{BE}$ represents the value of backoff delay and every device must cater this backoff delay (waiting time period in slots) before the channel access attempt. CW defines the number of backoff periods where the channel must be sensed to be idle before the start of transmission. CW implies after successful channel access attempt. NB determines the number of channel access attempts.

3.2. IEEE 802.15.6. IEEE 802.15.6 provides specification for the MAC layer to access the channel. The coordinator divides the channel into superframe time structures to allocate resources. Superframes are bounded by equal length beacons through the coordinator. Usually beacons are sent at beacon periods except inactive superframes or limited by regulation. The standard works under the following three channel access modes.

3.2.1. Beacon Mode with Beacon Period Superframe Boundaries. Beacons are sent at beacon periods by the coordinator and the superframe structure is managed by the coordinator using beacon frames. Figure 6 shows the frame structure for IEEE 802.15.6 [21].

The superframe structure consists of several phases: exclusive access phase 1 (EAP 1), random access phase 1 (RAP 1), type I/II phase, an EAP 2, RAP 2, and contention access phase (CAP). CSMA/CA or slotted Aloha is used by EAPs, RAPs, and CAPs. For emergency services and high priority data EAP 1 and EAP 2 are used, whereas CAP, RAP 1, and RAP 2 are used for regular data traffic. Types I/II are used for bilink allocation intervals, up-link and down-link allocation intervals, and delay bilink intervals. For resource allocation, the type I/II polling is used.

Figure 7 shows the CSMA/CA mechanism of this mode. A node's backoff counter value is set to a random integer number within [1, CW], where CW (the default value is $\mathrm{CW}_{\min }$ ) belongs to $\mathrm{CW}_{\min }$ and $\mathrm{CW}_{\max }$ which is dependent on user priority values given in Table 1 . When the algorithm starts, the node begins to counter decrement by one for the idle CSMA/CA slot duration (slot duration CSMA/CA (pCSMA/CA) slot length). A node is considered as CSMA/CA slot idle if the channel is idle between the start of slot and slot duration clear channel assessment (pCCATime). When the backoff counter reaches zero, the node transmits the data frame. If the case channel is busy because of some other frame transmission, then the node locks for its backoff counter until the channel gets idle. The value of CW gets double in case of an even number of failures until it reaches $\mathrm{CW}_{\max }[1,3,21]$. 


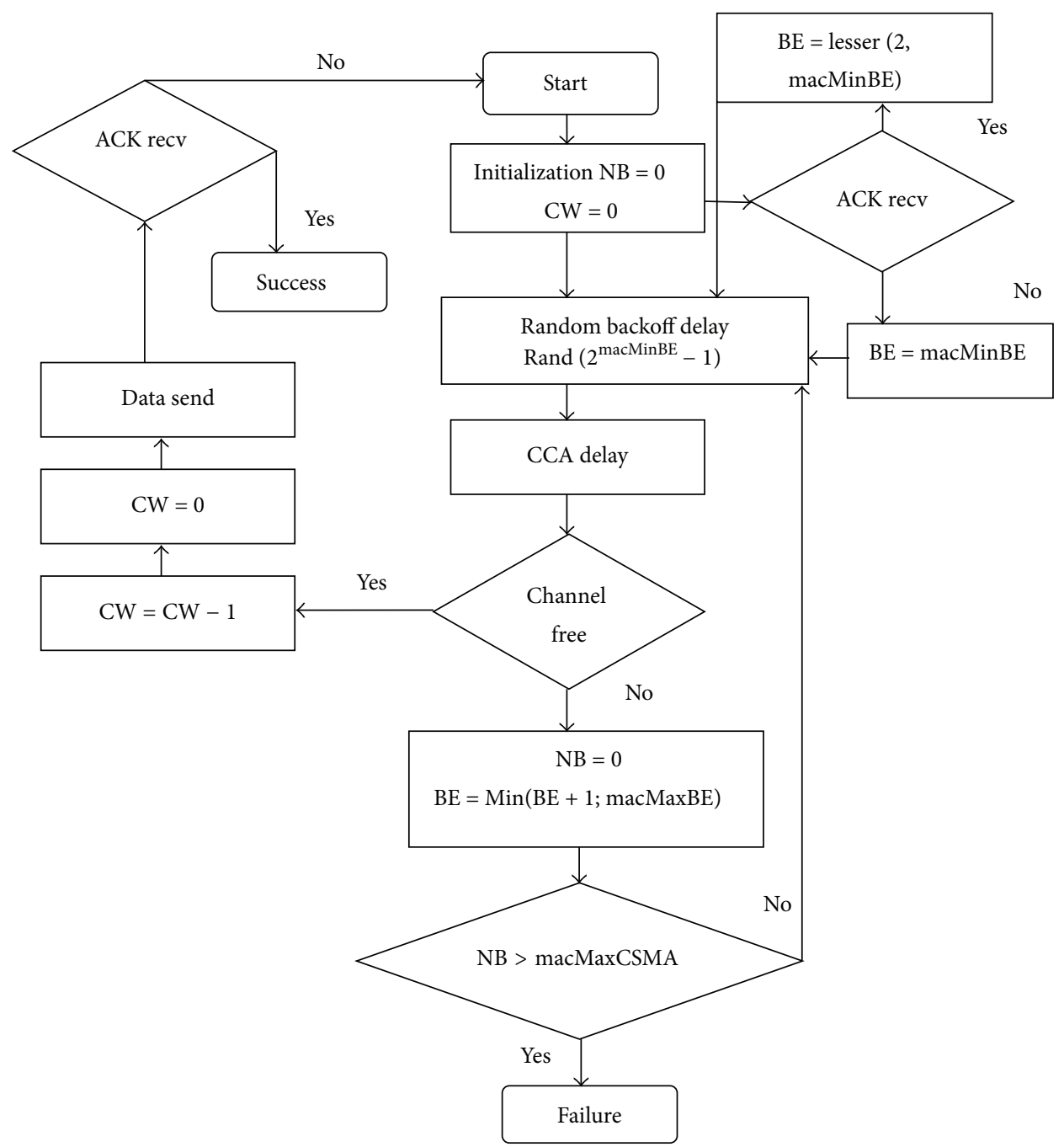

FIGURE 5: Data transmission flow chart for beacon mode of IEEE 802.15.4.

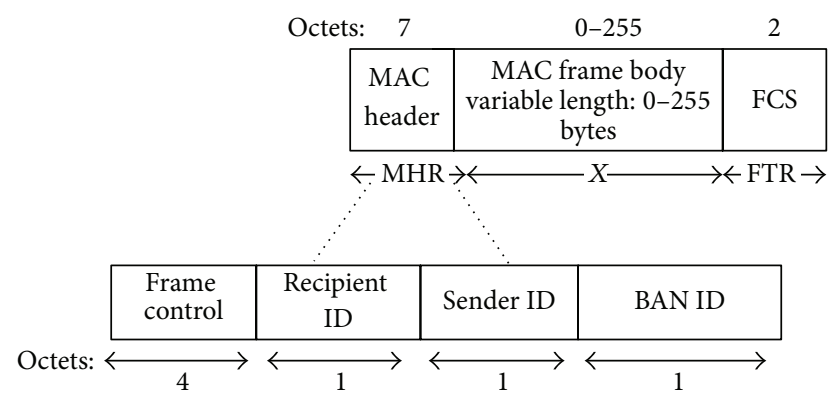

FIGURE 6: Frame structure of IEEE 802.15.6.

3.2.2. Nonbeacon Mode with Superframe Boundaries. In this channel access mode, beacons are not transmitted and channel is assigned by using polling mechanism.

3.2.3. Nonbeacon Mode without Superframe Boundaries. For this mode, the coordinator provides an unscheduled polled allocation and each node establishes its own schedule. Different access mechanisms are used in superframe phases: schedule access (connection oriented and contention-free access), improvised and unscheduled access (connectionless and contention-free access), and random access (CSMA/CA or slotted Aloha based).

\section{DRT Profile}

In this section, the DRT profile is computed after series of statistical experiments with the aim of performance optimization at the MAC layer for WBASN medical applications. The end-to-end delay model of IEEE 802.15.4 and IEEE 802.15.6 is used. Here, we will only consider the most significant values of the DRT profile which provides maximum reliability of data transmission within an acceptable latency and data rates. The word profile is used because it refers to a combination set of QoS parameters. The traditional profile of IEEE 802.15.4 imposes restrictions by giving default values for these MAC layer parameters as discussed in the previous section. The DRT focuses on CSMA/CA mechanisms of IEEE 802.15.4 


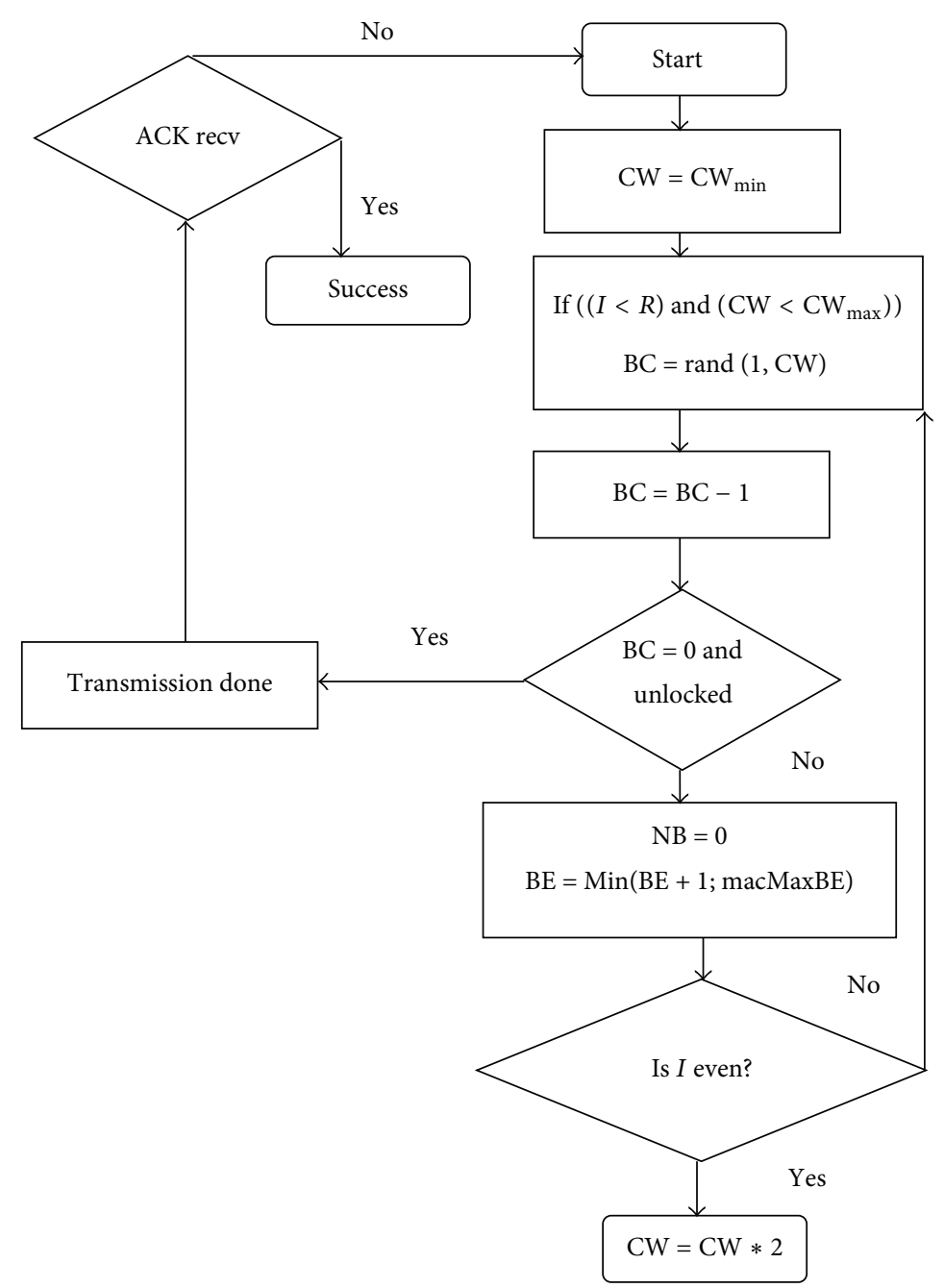

FIGURE 7: Data transmission flow chart for CSMA/CA in IEEE 802.15.6.

and IEEE 802.15.6 through CSMA/CA mechanisms, that is, CSMA/CA backoffs, backoff retries, and retransmissions.

The numerical formulas help to drive flexible and reliable set of combinational values which provide maximum reliability of data transmission within an acceptable latency and data rates. For IEEE 802.15.4, the DRT profile is defined for beaconless and beacon-enabled modes and it computes the value of the end-to-end delay with maximum reliability; for this case we refer macCSMABackoff and macFrameRetries as a reliability factor. The DRT provides those high reliability values that are within acceptable time boundaries for patient monitoring systems.

The following terminologies are used in the end-toend model which is modified in this research for medical applications:

ED is Expected Total Delay.

BPC is backoff time period including CCA.

CCA is clear channel assessment.

$T_{\text {frame }}$ is tansmission time for a frame with payload length.
$T_{\tau T}$ is turnaround time that is the time between a data frame.

$T_{\text {Ack }}$ is transmission time of an acknowledgment.

$T_{\text {SIFS }}$ is time for Short Interframe Space.

$N_{\text {BO }}$ is number of backoffs in one slot.

$T_{\text {BOslot }}$ is time for backoff slot.

$T_{\mathrm{CW}}$ is backoff period for contention window.

$\mathrm{ED}_{\mathrm{BC} 213}$ is end to end delay,

where $\mathrm{BE}$ is 2 , macCSMABackoff is 1 , and macMaxFrameRetries is 3 .

Using the above notations, we propose the modified equations from [21] below:

$$
\begin{aligned}
\mathrm{ED}= & \text { macFrameRetries } \\
& *\left(\mathrm{BPC}+T_{\text {frame }}+T_{\tau A}+T_{\mathrm{Ack}}+T_{\mathrm{SIFS}}\right), \\
\mathrm{BPC}= & \text { macCSMABackoff } *\left(N_{\mathrm{BO}} * T_{\mathrm{BOslot}}+\mathrm{CCA}\right) .
\end{aligned}
$$


$N_{\mathrm{BO}}$ is a random value of time:

$$
\begin{aligned}
& N_{\text {BO }}=\left(2^{\text {macMinBE }}-1\right), \\
& T_{\text {BOslot }}=20 * T_{\text {symbol }}=\frac{20 * 1}{\text { Bitrate }}, \\
& \text { CCA }=8 * T_{\text {symbol }}=0.000390 \mathrm{sec}=0.390 \mathrm{~ms}, \\
& T_{\text {frame }} \\
& \quad=\frac{(\text { PHY Header }(\text { in bits })+\text { MAC header (in bits }))}{\text { Data rate }} .
\end{aligned}
$$

For example, if we send a maximum payload size then $T_{\text {frame }}$ is

$$
\begin{aligned}
T_{\text {frame }} & =133 * \frac{8}{20 * 1024}=51.95 \mathrm{~ms}, \\
T_{\tau \mathrm{A}} & =12 * T_{\text {symbol }}, \\
T_{\text {Ack }} & =\frac{31 * 8}{20 * 1024}=12.1 \mathrm{~ms}, \\
T_{\text {SIFS }} & =12 * T_{\text {symbol }} .
\end{aligned}
$$

$\mathrm{ED}$ in (3) represents an end-to-end delay in the CSMA/CA process in the beaconless/unslotted mode. macFrameRetries represents the number of retransmission attempts when the MAC layer does not receive acknowledgement. Its default value is 4 and the maximum value is 7. BPC represents the backoff time period which will be assigned to a node before going to channel access attempt. CCA is the clear channel assessment time period. We add this value with the backoff time period in (4), so every time when a node senses the channel, it must wait for two time periods, that is, backoff and CCA. $N_{\mathrm{BO}}$ represents the number of backoffs (a specific time period) in one slot. $T_{\text {BOslot }}$ represents the time for a backoff slot. For analysis, we consider the bit rate of $20 \mathrm{kbps}$ and $250 \mathrm{kbps}(868.0-868.6 \mathrm{MHz}$ and $2.4 \mathrm{GHz}$ frequency band are considered with BPSK modulation and a symbol represents 1 and 4 bits). The output of the DRT profile is represented in Tables 5(a) and 5(b).

From a series of experiments, several significant values for the DRT profile are selected and recommended for WBASNs in medical applications. Tables 5(a) and 5(b) describe suitable combination (the combinations that provide maximum reliability within $250 \mathrm{~ms}$ ) of the DRT profile computed values after statistical experiments with different frequencies and the data rate by considering the maximum packet size, that is, 127 bytes in IEEE 802.15.4.

Similarly, (7) represents the end-to-end delay for IEEE 802.15.4 beacon-enabled. The difference between the IEEE 802.15.4 beacon mode (slotted CSMA/CA) and the nonbeacon mode (unslotted CSMA/CA) is the addition of $T_{\mathrm{CW}}$ which represents the part of the backoff time for the slotted CSMA/CA in the algorithm with contention window (CW). Table 6 represents significant (containing only those reliable
TABLE 5: (a) End-to-end delay in the beaconless mode for $868 \mathrm{MHz}$. (b) End-to-end delay in the beaconless mode for $2.4 \mathrm{GHz}$.

(a)

\begin{tabular}{lc}
\hline $\begin{array}{l}\text { Few DRT-combinations of reliability and delay sensitive } \\
\text { applications for } 868 \mathrm{MHz} \text { and } 20 \mathrm{kbps} \text { for the nonbeacon mode } \\
\text { Parameter combination }\end{array}$ & ED delay (ms) \\
\hline $\mathrm{ED}_{253}$ & 222.072 \\
$\mathrm{ED}_{352}$ & 168.7 \\
$\mathrm{ED}_{333}$ & 237.8 \\
$\mathrm{ED}_{314}$ & 258 \\
$\mathrm{ED}_{442}$ & 235 \\
$\mathrm{ED}_{413}$ & 217 \\
$\mathrm{ED}_{423}$ & 262 \\
$\mathrm{ED}_{522}$ & 237 \\
$\mathrm{ED}_{557}$ & 1474 \\
\hline
\end{tabular}

(b)

DRT-combination of reliability and delay sensitive applications for $2.4 \mathrm{GHz}$ and $250 \mathrm{kbps}$ for the nonbeacon mode IEEE 802.15.4

\begin{tabular}{lc} 
Parameter combination & ED delay $(\mathrm{ms})$ \\
\hline $\mathrm{ED}_{212}$ & 13 \\
$\mathrm{ED}_{375}$ & 108 \\
$\mathrm{ED}_{473}$ & 117 \\
$\mathrm{ED}_{475}$ & 195 \\
$\mathrm{ED}_{545}$ & 223 \\
$\mathrm{ED}_{573}$ & 222 \\
$\mathrm{ED}_{625}$ & 225 \\
$\mathrm{ED}_{643}$ & 250 \\
$\mathrm{ED}_{662}$ & 248 \\
\hline
\end{tabular}

values which shows latency within $250 \mathrm{~ms}$ ) statistical experiment results of the beacon mode. Consider

$$
\begin{aligned}
\mathrm{ED}= & \text { macFrameRetries } \\
& *\left(\mathrm{BPC}+T_{\text {frame }}+T_{\tau r}+T_{\mathrm{Ack}}+T_{\mathrm{SIFS}}+T_{\mathrm{CW}}\right),
\end{aligned}
$$

where $T_{\mathrm{CW}}=7.8125 \mathrm{~ms}$.

Similarly, (8) represents the end-to-end delay for IEEE 802.15.6 with different priority values. In (9), to compute the value of $T_{\mathrm{CW}}$, we consider the average values of $\mathrm{CW}_{\min }, \mathrm{CW}_{\max }$. Consider

$$
\begin{aligned}
\mathrm{ED}= & \text { macMaxFrameRetries } \\
& *\left(T_{\mathrm{CW}}+T_{\text {frame }}+T_{\tau r}+T_{\text {Ack }}\right), \\
T_{\mathrm{CW}}= & \operatorname{Avg}\left(\mathrm{CW}_{\min }, \mathrm{CW}_{\max }\right) *\left(T_{\mathrm{CCA}}+20 \mu \mathrm{s}\right) .
\end{aligned}
$$

We consider Data rate of $75.9 \mathrm{kbps}$ and $250 \mathrm{kbps}$ with symbol rate (Rs) of $187.5 \mathrm{kbps}$ and $600 \mathrm{kbps}$, respectively. Consider

$$
T_{\mathrm{CCA}}=\frac{63}{\mathrm{Rs}} .
$$


TABLE 6: DRT profile values for the IEEE 802.15 .4 beacon-enabled mode.

\begin{tabular}{lc}
\hline $\begin{array}{l}\text { Suitable combination for reliability and delay sensitive } \\
\text { applications (with retransmission) for } 2.4 \mathrm{GHz} \text { and } 250 \mathrm{kbps} \text { for } \\
\text { the beacon mode }\end{array}$ \\
Parameter combination & ED delay (ms) \\
\hline $\mathrm{ED}_{212}$ & 29 \\
$\mathrm{ED}_{272}$ & 42 \\
$\mathrm{ED}_{473}$ & 141 \\
$\mathrm{ED}_{475}$ & 235 \\
$\mathrm{ED}_{535}$ & 213 \\
$\mathrm{ED}_{572}$ & 164 \\
$\mathrm{ED}_{573}$ & 246 \\
$\mathrm{ED}_{652}$ & 224 \\
$\mathrm{ED}_{633}$ & 218 \\
\hline
\end{tabular}

In (10), we consider two different values for $T_{\mathrm{CCA}}$, that is, $0.329 \mathrm{~ms}$ for $420-450 \mathrm{MHz}$ frequency band and $0.102 \mathrm{~ms}$ for $2.4 \mathrm{GHz}$ frequency band. $T_{\tau A}$ represents the turnaround time, which is the elapsed time from the end of the received frame to the start of the transmitted frame. This should be between duration of short interframe spacing (pSIFS) and pSIFS+ slot duration extrainterframe spacing (pExtraIFS), the value in the worst case:

$$
\begin{aligned}
T_{\tau A} & =\text { pSIFS }+ \text { pExtraIFS }=85 \mu \mathrm{s}=0.085 \mathrm{~ms} \\
T_{\text {Ack }} & =\frac{(9 * 8)+31}{\text { Data rate }}=\frac{103}{75.9} * 1024=1.32 \mathrm{~ms} .
\end{aligned}
$$

Table 7 describes several suitable combinations of the DRT profile computed through statistical experiments with $420-450 \mathrm{MHz}$ with data rate $75.9 \mathrm{kbps}$ by considering the maximum packet size, that is, 254 bytes in IEEE 802.15.6.

\section{Validation and Simulation}

Open source Castalia 3.2 is used as the simulator in this research. Moreover at the application layer, the throughput test application is used which is integrated with the $\mathrm{OMNeT}++$ simulator. In the simulated scenario, all transmitting nodes send packet to a central hub (node 0 ). Table 8 shows the simulation parameters. This section contains three steps below:

(I) Validation of the proposed DRT profile by comparing statistical results of maximum throughput (MT) with the MT values defined by IEEE 802.15.4 and IEEE 802.15.6 standards.

(II) Comparison in terms of packet delivery ratio (PDR) of the proposed DRT profile values with the existing profile (the standard values of CSMA/CA used by simulators and standards).

(III) Delay computation of the DRT profile with different packet sizes to check the performance and usability of DRT for different medical applications.

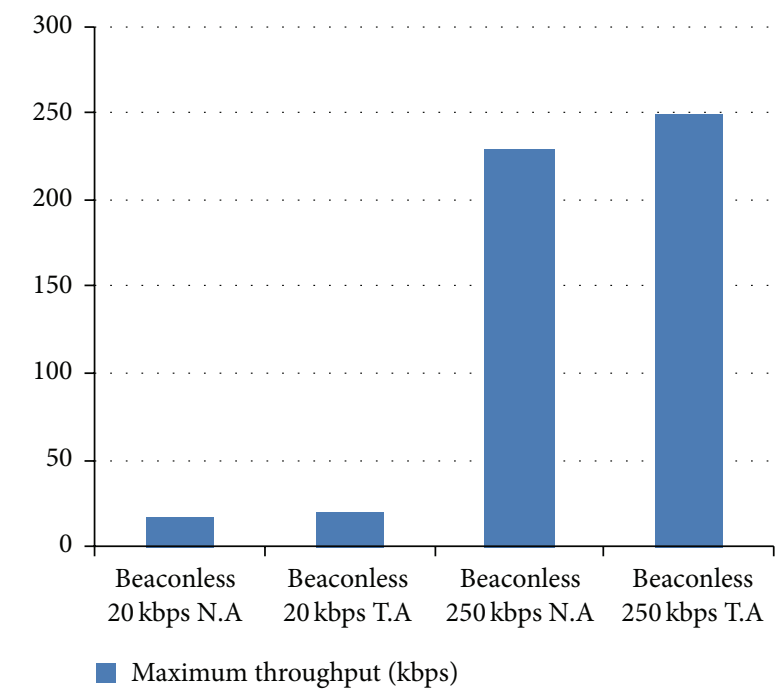

FIGURE 8: MT numerical and theoretical comparison of the IEEE 802.15.4 beaconless (unslotted) modes.

5.1. Validation of the Proposed DRT Profile by Comparing Statistical Results of Maximum Throughput (MT) with the MT Values Defined by IEEE 802.15.4 and IEEE 802.15.6 Standards. The presented numerical and statistical model is validated by comparing the MT values for numerical (values through statistical equations described above) and theoretical (those values which standard document presents) values under different frequency bands and data rates of IEEE 802.15.4 and IEEE 802.15.6. MT refers to a number of MAC Layer Service Data Units (MSDUs) in a unit time. Each MSDU involves overhead at MAC and PHY layers including headers, control frames, interframe spacing, and backoff. The MT is defined as a ratio of giving payload size $x$ (bytes) to the total transmission delay for specific payload size $x$ :

$$
\text { Maximum throughput }(\mathrm{MT})=\frac{8 * x}{\operatorname{ED}(x)} \text {. }
$$

The following assumptions are used while conducting simulations under step (I):

(i) The node always continuously trying to send a packet.

(ii) Perfect channel conditions.

(iii) Zero bit error rate (BER).

(iv) One sender and one receiver.

Figures 8, 9, and 10 show the comparative MT analysis between numerical analysis (N.A) and theoretical analysis (T.A) values of IEEE 802.15.4 and IEEE 802.15.6 under different frequency bands and data rates. Figure 8 describes MT comparison of the IEEE 802.15.4 beaconless modes under frequency bands, that is, $868 \mathrm{MHz}$ (European licensed band) and $2.4 \mathrm{GHz}$ (ISM band).

Theoretically in ideal conditions $868 \mathrm{MHz}$ provides a data rate of $20 \mathrm{kbps}$, whereas $2.4 \mathrm{GHz}$ provides up to $250 \mathrm{kbps}$ depending on the symbol rate of the underlying modulation scheme. The N.A values are close to T.A values, that is, 
TABLE 7: Significant DRT profile values for IEEE 802.15.6.

\begin{tabular}{|c|c|c|c|c|c|c|c|c|c|c|}
\hline \multirow{2}{*}{ User priority } & \multirow{2}{*}{$\mathrm{CW}_{\min }$} & \multirow{2}{*}{$\mathrm{CW}_{\max }$} & \multirow{2}{*}{$T_{\mathrm{CW}}(\mathrm{ms})$} & \multirow{2}{*}{$\mathrm{ED}(\mathrm{ms})$} & \multicolumn{6}{|c|}{ ED values with different retransmission values } \\
\hline & & & & & 2 & 3 & 4 & 5 & 6 & 7 \\
\hline 0 & 16 & 64 & 10.92 & 21 & 42 & 63 & 84 & 105 & 126 & 147 \\
\hline 1 & 16 & 32 & 6.552 & 16.6 & 33.2 & 49.8 & 66.4 & 83 & 99.6 & 116.2 \\
\hline 2 & 8 & 32 & 5.46 & 15.5 & 31 & 46.5 & 66 & 77.5 & 93 & 108.5 \\
\hline 3 & 8 & 16 & 3.27 & 13.8 & 27.6 & 41.4 & 55.2 & 69 & 83 & 96.6 \\
\hline 4 & 4 & 16 & 2.73 & 12.74 & 25.4 & 38.1 & 51 & 64 & 77 & 88.9 \\
\hline 5 & 4 & 8 & 1.638 & 11.67 & 23.34 & 35.01 & 46.68 & 58.35 & 70.2 & 81.6 \\
\hline 6 & 2 & 8 & 1.365 & 11.37 & 22.7 & 34 & 45.3 & 56.5 & 67.8 & 80.2 \\
\hline 7 & 1 & 4 & 0.81 & 10.81 & 21.6 & 32.5 & 45 & 49 & 64 & 75.6 \\
\hline
\end{tabular}

TABLE 8: Simulation setup.

\begin{tabular}{lc}
\hline Parameters & Value \\
\hline Number of nodes & $6-20$ \\
MAC & IEEE 802.15 .4 , IEEE 802.15 .6 \\
Channel mode & Log shadowing wireless model \\
Path loss exponent & 2.4 \\
Simulation time & $5-20$ minutes \\
Seed value & 11 \\
Frequencies band & $420 \mathrm{MHz}, 868 \mathrm{MHz}, 2.4 \mathrm{GHz}$ \\
Data rates & $20 \mathrm{kbps}, 75.9 \mathrm{kbps}, 242.9 \mathrm{kbps}$, \\
& $250 \mathrm{kbps}$ \\
Evaluation parameters & Delay, packet delivery ratio \\
Considered variations & (PDR), throughput \\
\hline
\end{tabular}

$17 \mathrm{kbps}$ for $868 \mathrm{MHz}$ and $235 \mathrm{kbps}$ for $2.4 \mathrm{GHz}$. Figure 9 demonstrates the result in the same pattern as described in Figure 8. In Figure 10, we consider two frequency bands $420 \mathrm{MHz}$ (for implanted body sensors) with $75.9 \mathrm{kbps}$ and $2.4 \mathrm{GHz}$ with $242.9 \mathrm{kbps}$ depending on the used modulation scheme. N.A analysis values are close to actual theoretical values, that is, $60 \mathrm{kbps}$ and $225 \mathrm{kbps}$. The results of the 1st step validate the proposed numerical model and in the 2 nd step extensive simulation is conducted by considering different aspects of WBASNs.

5.2. Comparison in terms of Packet Delivery Ratio (PDR) of the Proposed DRT Profile with the Existing Profile (Standard Values of CSMA/CA Used by Simulators and Standards). Figures 11 and 12 show the PDR values comparison between the default standard profile and the different DRT profiles values for IEEE 802.15.4 and IEEE 802.15.6.

The significant improvement is observed for the DRT profile values as the default profile provides 65\% PDR, whereas the DRT profile values provide up to $90 \%$. The reason for the improvement is the selection of maximum reliability within a limited time period. Figure 11 shows the PDR values comparison among the default standard profile and the different DRT profiles values for IEEE 802.15.6. IEEE

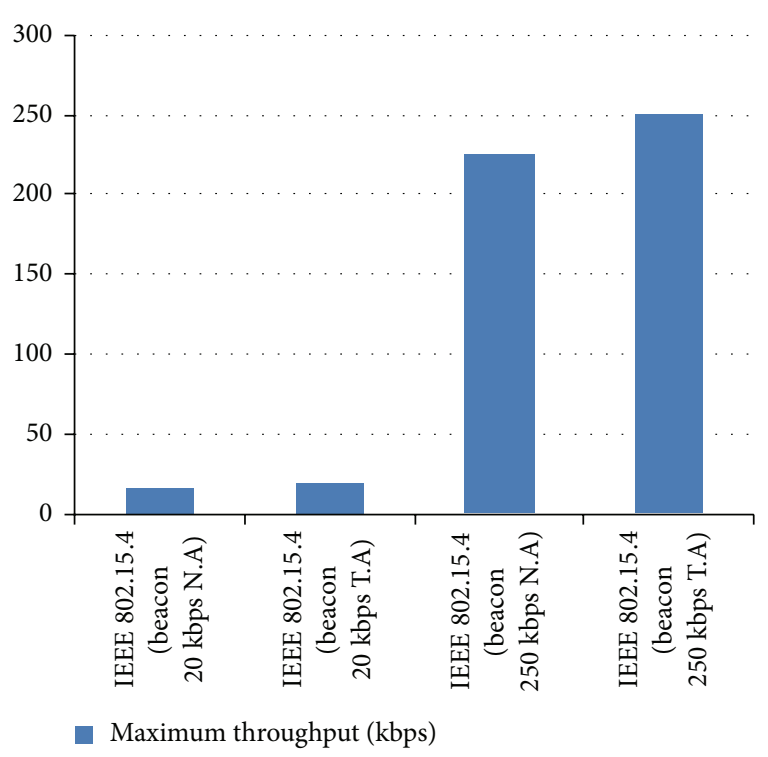

FIGURE 9: MT numerical and theoretical comparison of the IEEE 802.15.4 beacon-enabled modes.

802.15.6 provides seven priority classes where seven is the highest and zero is the lowest priority class. Figure 12 shows the comparison result of priority 3 class and priority 7 class with their default and DRT values. It is observed that priority classes with the DRT profile provide better PDR values; that is, for priority 3 with the default MAC parameter settings the PDR is $67 \%$, whereas for the DRT profile the PDR is $84 \%$. Similarly, for the default profile with priority 7 the PDR is $77 \%$, whereas with the DRT profile the PDR is $90 \%$. The difference between the PDR values is due to improved reliability values under the DRT profile.

5.3. Delay Computation of the DRT Profile with Different Packet Sizes to Check the Performance and Usability of DRT for Different Medical Applications. After proving that the DRT profile performs better in terms of packet reception, we evaluate and validate the DRT profile values for latency (we compare statistical results with simulation results) in real environments by considering the different packet size of 


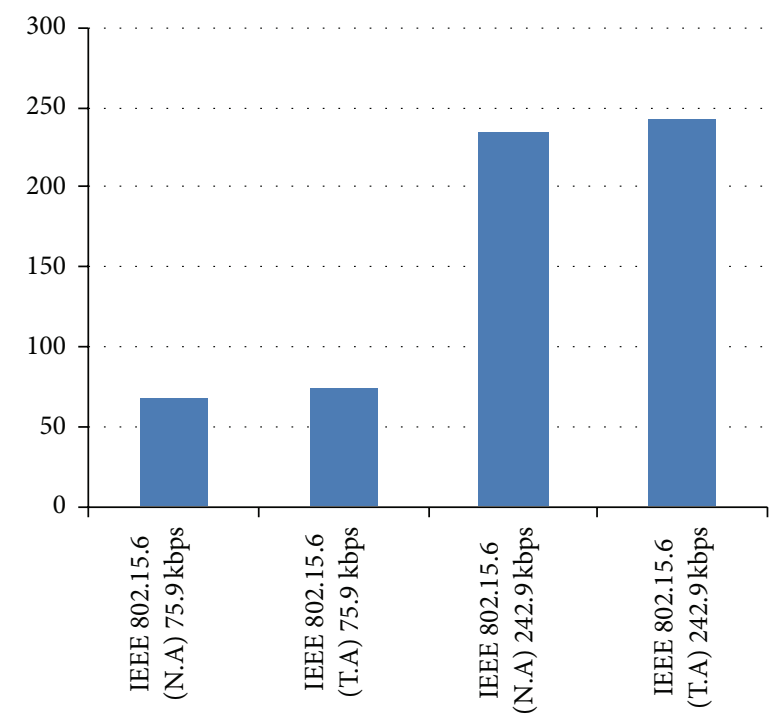

Maximum throughput (kbps)

FIgURE 10: MT numerical and theoretical comparison of IEEE 802.15.6.

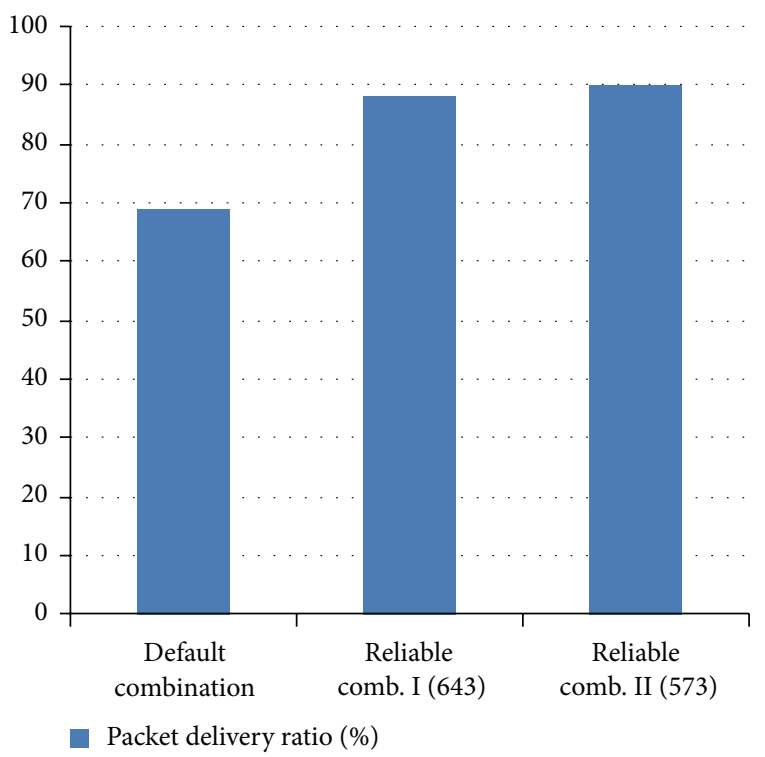

FIGURE 11: Packet delivery ratio (PDR) comparison between the default profile and the DRT profile for IEEE 802.15.4.

various WBASNs applications with different number of nodes in a star topology. The purpose of packet size variation is to align our research with various biomedical sensor network requirements; for example, an ECG node requires $8 \mathrm{kbps}$, a blood pressure device requires $960 \mathrm{bps}$, a temperature node requires $320 \mathrm{bps}$, and so forth. These simulations focus on analyzing the performance of different evaluating parameters like packet delivery ratio (PDR) and latency for WBASNs applications under different packet sizes (as each sensor has its own packet size requirement).

Latency is defined as arriving message time minus the message sending time, whereas PDR is defined as the ratio of

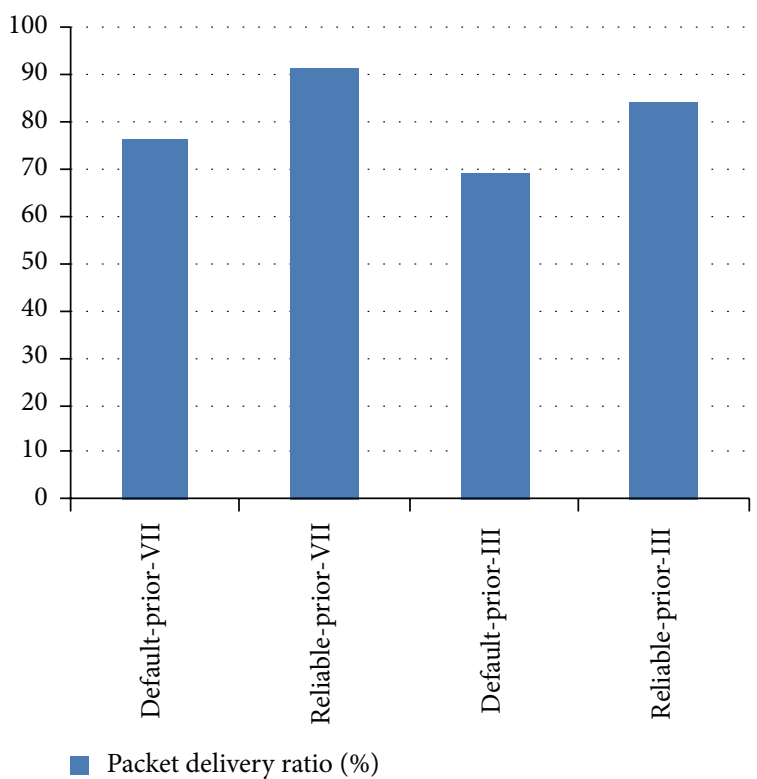

FIGURE 12: Packet delivery ratio (PDR) comparison between the default profile and the DRT profile for IEEE 802.15.6.

the number of successful delivered packets to a node to total number of transmitting data packets to a node.

Table 1 shows that most of the WBASNs applications required time bounded services in form of specific latency. $250 \mathrm{~ms}$ is the maximum reasonable latency value which will be used as a benchmark value for evaluations. In simulations, we choose several significant DRT profile values which provide maximum reliability within $250 \mathrm{~ms}$. The following assumptions are used while conducting simulations under step (II):

(i) The node always continuously trying to send a packet.

(ii) Normal channel conditions (not ideal).

(iii) Multiple senders and one receiver.

Figures 13 and 14 show the delay comparison between N.A and S.A of the different DRT profiles values for IEEE 802.15.4 (beacon-enabled with $20 \mathrm{kbps}(868 \mathrm{MHz})$ and $250 \mathrm{kbps}(2.4 \mathrm{GHz})$ ). The delay is computed for different packet sizes up to the maximum packet size, that is, 127 bytes in IEEE 802.15.4. Overall, delay increases with the increase of the packet size, but it remains around $250 \mathrm{~ms}$ for the DRT profile.

It is observed that delay values of the S.A curve are higher than the N.A values. This is obvious; as the number of nodes increases, the collision probability also increases, which results in higher delay values. Different frequencies band and data rate for the same DRT profile give different values of delay. Usually, higher frequency with $250 \mathrm{kbps}$ provides lesser delay values as compared with a low frequency band. This is because higher data rates offer more bandwidth to the data traffic, which produces less delay.

Figures 15 and 16 demonstrate the delay comparison between N.A and S.A of the different DRT profiles values for IEEE 802.15.4 (beaconless with $20 \mathrm{kbps}$ and $250 \mathrm{kbps}$ ). 


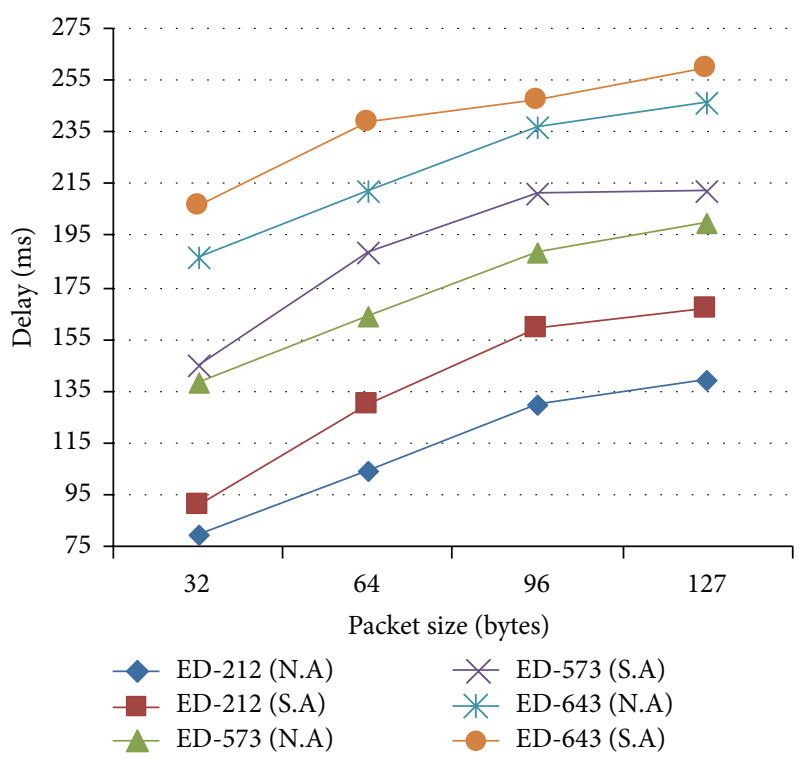

FIGURE 13: Delay comparison of different MAC layer combinations for IEEE 802.15.4 (beacon-enabled, $20 \mathrm{kbps}$ ).

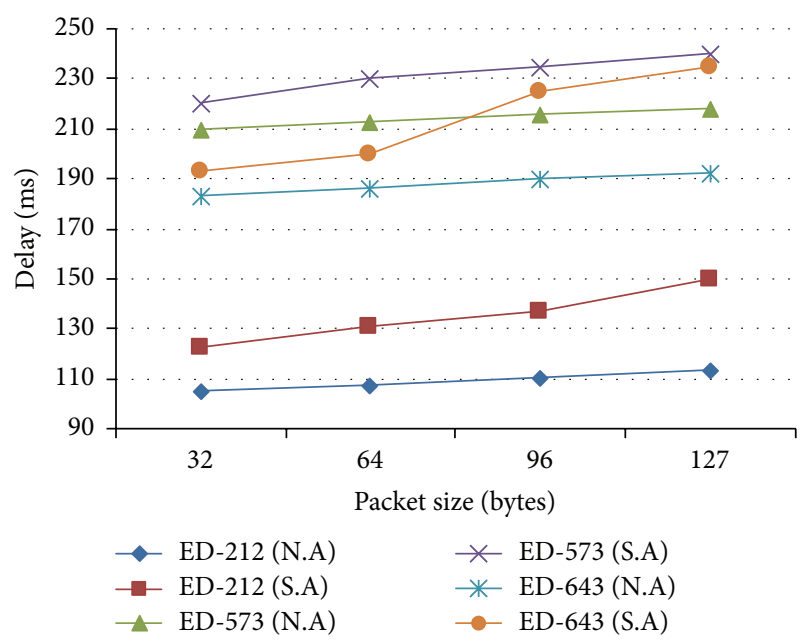

FIGURE 14: Delay comparison of different combinations of IEEE 802.15.4 (beacon-enabled, $250 \mathrm{kbps).}$

Overall, delay increases with the increase of packet size but it remains around $250 \mathrm{~ms}$ for the DRT profile. The DRT profile values are different for beaconless and beacon-enabled modes. It is observed that the beaconless mode provides less delay than the beacon-enabled mode. It is also observed that delay values of the S.A curve are higher than the N.A values. The reason is that as the number of nodes increases, the channel access attempts will also increase; only one node gets the channel and others have to wait till the next channel access; this wait increases the end-to-end delay.

Figures 17 and 18 show the delay comparison between N.A and S.A of the different DRT profiles values under different user priorities for IEEE 802.15.6 (with $75.9 \mathrm{kbps}$ $(420 \mathrm{MHz})$ and $242.9 \mathrm{kbps}(2.4 \mathrm{GHz}))$. The delay is computed for different packet sizes up; overall, the delay increases with

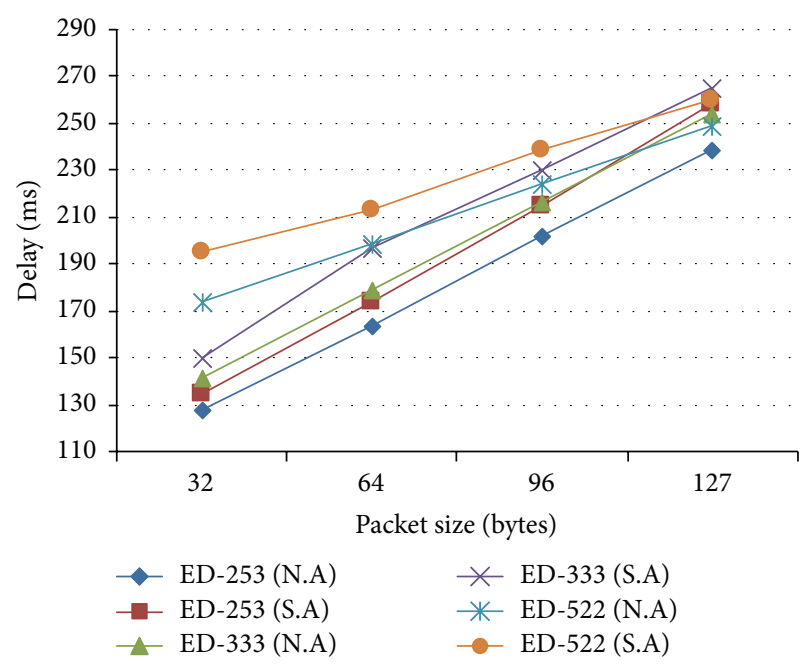

FIGURE 15: Delay comparison of different MAC layer combinations (beaconless, $20 \mathrm{kbps}$ ).

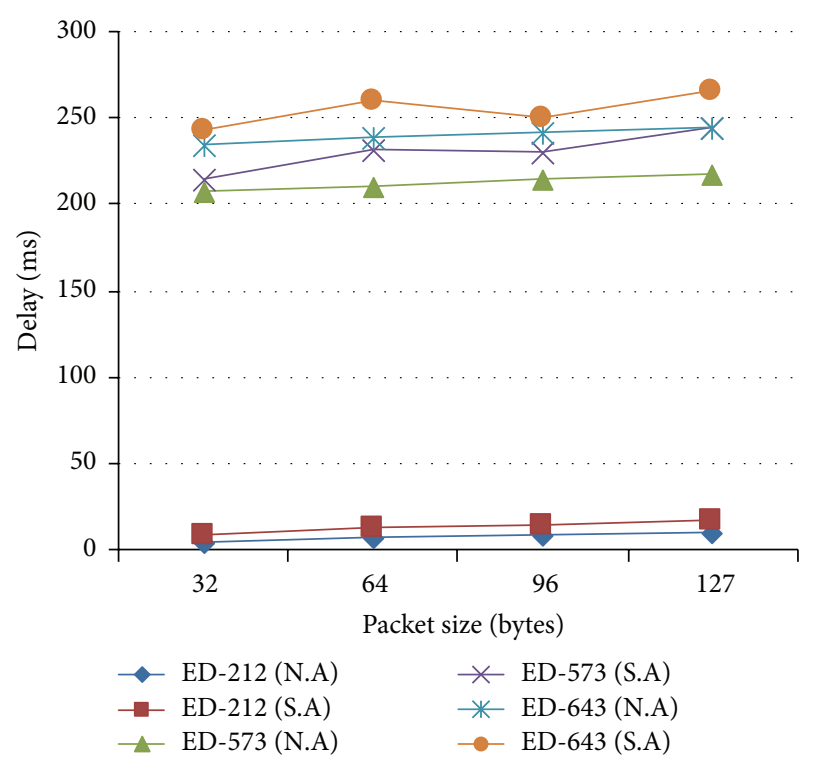

FIGURE 16: Delay comparison of different combinations of IEEE 802.15.4 (beaconless, $250 \mathrm{kbps}$ ).

the increase of packet size, but it remains around $250 \mathrm{~ms}$ for the DRT profile. It is observed that delay values of the S.A curve are higher than the N.A values. The difference in delay values between priority 3 and priority 7 is observed. Priority 7 provides less delay because it gets earlier and more channel opportunities than priority 3 . Moreover, as the number of nodes increases, the collision probability also increases, which results in higher delay values. Different frequencies band and data rate for the same DRT profile give different values of delay. Usually, higher frequency with $250 \mathrm{kbps}$ provides lesser delay values as compared with a low frequency band. This is because higher data rates offer more bandwidth to data traffic, which produces less delay. 


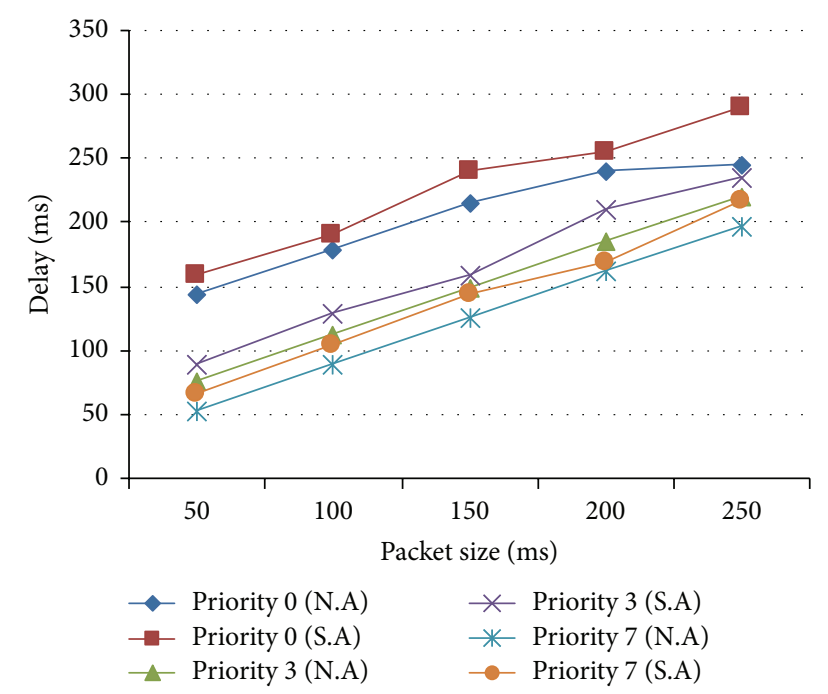

FIGURE 17: Delay comparison of different priority classes of IEEE 802.15.6 (75.9 kbps).

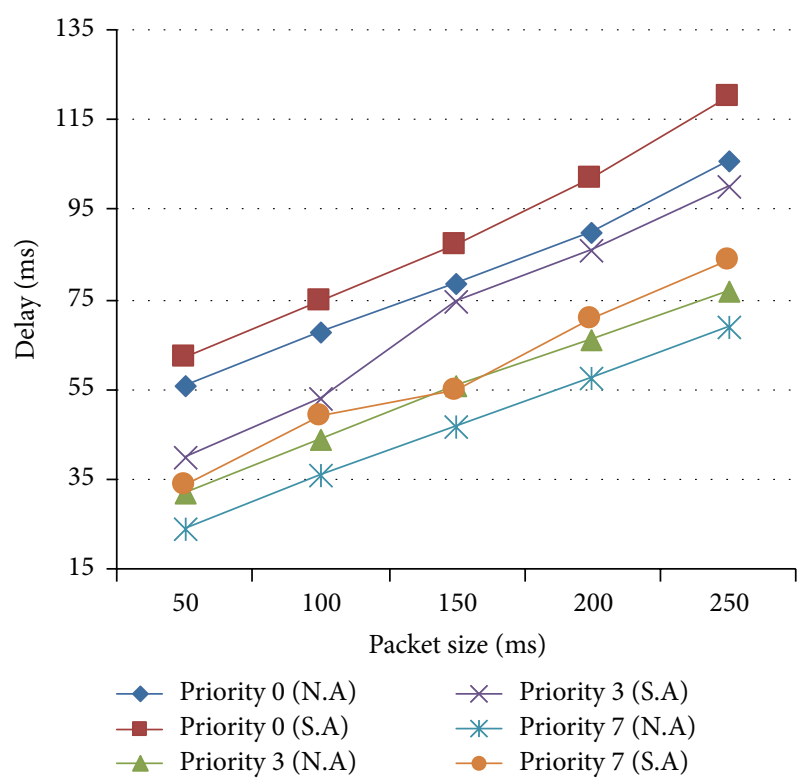

FIGURE 18: Delay comparison of different priority classes of IEEE 802.15.6 (242.9 kbps).

\section{Conclusion}

WBASN provides new opportunities for the deployment of a range of medical applications including patient monitoring and activity recognition. WBASN imposes a specific set of QoS requirements which are necessary for developing WBASN MAC protocols. These QoS requirements for WBASN have been highlighted in this paper. Furthermore, we have explored IEEE 802.15.4 and IEEE 802.15.6 based WBASN MAC protocols specifically for medical applications and investigated the various optimization mechanisms on these protocols. Although these optimized solutions provide better QoS, they lack a set of QoS requirements at a given time. In this context, the DRT profile has been proposed on the basis of the end-to-end delay model with the purpose of maximum reliability of data transmission within an acceptable latency and data rates. The numerical model has been validated in $\mathrm{OMNeT}++$ with the Castalia 3.2 simulation tool. Moreover, comprehensive set of simulations has been conducted in evaluating the end-to-end delay, PDR, and MT by varying the packet size, number of nodes, frequency bands, and data rates. The results have shown that optimization in delay and PDR could be achieved by applying the DRT profile instead of a standard parameter profile. We have also concluded that PHY standards with higher data rates and high frequencies could provide a less delay value at the MAC layer as compared to low frequency and low data rates. The purpose of applying different packet sizes is to provide IEEE 802.15.4 and IEEE 802.15.6 CSMA/CA MAC suitability with various WBASNs medical applications as these applications demand maximum reliability within specific delay limits. An acceptable gap, which is due to collisions and channel occupancy of different nodes, has been also observed between numerical and simulated results. We believe that the conclusions and experimental results obtained in this research can be helpful for protocol designers and researchers to establish optimized MAC protocols and standards for a variety of medical applications.

\section{Conflict of Interests}

There is no conflict of interests among the authors about this research.

\section{References}

[1] S. Movassaghi, M. Abolhasan, J. Lipman, D. Smith, and A. Jamalipour, "Wireless body area networks: a survey," IEEE Communications Surveys \& Tutorials, vol. 16, no. 3, pp. 16581686, 2014.

[2] M. Shu, D. Yuan, C. Zhang, Y. Wang, and C. Chen, "A MAC protocol for medical monitoring applications of wireless body area networks," Sensors, vol. 15, no. 6, pp. 12906-12931, 2015.

[3] R. Cavallari, F. Martelli, R. Rosini, C. Buratti, and R. Verdone, "A survey on wireless body area networks: technologies and design challenges," IEEE Communications Surveys \& Tutorials, vol. 16, no. 3, pp. 1635-1657, 2014.

[4] D. Barata, L. Gonçalo, C. Andreia, and D. António, "System of acquisition, transmission, storage and visualization of Pulse Oximeter and ECG data using Android and MQTT," Procedia Technology, vol. 9, pp. 1265-1272, 2013.

[5] J. Ahmad and Z. Fareeha, "Review of body area network technology \& wireless medical monitoring," International Journal of Information, vol. 2, no. 2, 2012.

[6] N. Bradai, L. Chaari, and L. Kamoun, "A comprehensive overview of wireless body area networks (WBAn)," in Digital Advances in Medicine, E-Health, and Communication Technologies, pp. 1-32, IGI Global, 2013.

[7] J. Valverde, V. Rosello, G. Mujica, J. Portilla, A. Uriarte, and T. Riesgo, "Wireless sensor network for environmental monitoring: application in a coffee factory," International Journal of Distributed Sensor Networks, vol. 2012, Article ID 638067, 18 pages, 2012. 
[8] P. Campbell, "Current population reports (population projections: states, 1995-2025)," Census Bureau, pp. 25-1131, 2013.

[9] H. C. Keong and M. R. Yuce, "UWB-WBAN sensor node design," in Proceedings of the Annual International Conference on Engineering in Medicine and Biology Society (EMBC '11), pp. 2176-2179, Boston, Mass, USA, August 2011.

[10] I. Beretta, F. Rincon, N. Khaled et al., "Model-based design for wireless body sensor network nodes," in Proceedings of the 13th IEEE Latin American Test Workshop (LATW'12), pp. 1-6, Quito, Ecuador, April 2012.

[11] K. M. S. Thotahewa, J. Y. Khan, and M. R. Yuce, "Power efficient ultra wide band based wireless body area networks with narrowband feedback path," IEEE Transactions on Mobile Computing, vol. 13, no. 8, pp. 1829-1842, 2014.

[12] F. Sun, Z. Zhao, Z. Fang, Y. DuLidong, H. Li, and L. Tian, "Design and implementation of a high integrated noncontact ECG monitoring node for wireless body sensor networks," in Ubiquitous Information Technologies and Applications, vol. 280 of Lecture Notes in Electrical Engineering, pp. 6335-641, Springer, 2014.

[13] S. M. Mahalle and P. V. Ingole, "Design and implementation of wireless body area sensor network based health monitoring system," International Journal of Engineering Research and Technology, vol. 2, no. 6, 2013.

[14] Y. Zhang, Y. Shakhsheer, A. T. Barth et al., "Energy efficient design for body sensor nodes," Journal of Low Power Electronics and Applications, vol. 1, no. 1, pp. 109-130, 2011.

[15] G. Tartarisco, G. Baldus, D. Corda et al., "Personal Health System architecture for stress monitoring and support to clinical decisions," Computer Communications, vol. 35, no. 11, pp. 12961305, 2012.

[16] R. Mumford, "Enhanced wireless technology for body implants and sensors," Microwave Journal, vol. 56, no. 9, p. 47, 2013.

[17] K. M. S. Thotahewa, J. Y. Khan, and M. R. Yuce, "Power efficient ultra wide band based wireless body area networks with narrowband feedback path," IEEE Transactions on Mobile Computing, vol. 13, no. 8, pp. 1829-1842, 2014.

[18] K. A. Al-Saud, M. Mahmuddin, and A. Mohamed, "Wireless body area sensor networks signal processing and communication framework: survey on sensing, communication technologies, delivery and feedback," Journal of Computer Science, vol. 8, no. 1, pp. 121-132, 2012.

[19] M. Sung, M. Carl, and P. Alex, "Hypoxia silences the neural activities in the early phase of the phrenic neurogram of eupnea in the piglet," Journal of NeuroEngineering and Rehabilitation, vol. 2, article 32, 2005.

[20] F. Xia, R. Hao, Y. Cao, and L. Xue, "A survey of adaptive and realtime protocols based on IEEE 802.15.4," International Journal of Distributed Sensor Networks, vol. 2011, Article ID 212737, 11 pages, 2011.

[21] N. Bradai, L. C. Fourati, and L. Kamoun, "Investigation and performance analysis of MAC protocols for WBAN networks," Journal of Network and Computer Applications, vol. 46, pp. 362$373,2014$.

[22] U. Anliker, J. A. Ward, P. Lukowicz et al., "AMON: a wearable multiparameter medical monitoring and alert system," IEEE Transactions on Information Technology in Biomedicine, vol. 8, no. 4, pp. 415-427, 2004.

[23] R. Paradiso, G. Loriga, and N. Taccini, "A wearable health care system based on knitted integrated sensors," IEEE Transactions on Information Technology in Biomedicine, vol. 9, no. 3, pp. 337344, 2005.
[24] A. Lymberis and R. Paradiso, "Smart fabrics and interactive textile enabling wearable personal applications: R\&D state of the art and future challenges," in Proceedings of the 30th Annual International Conference of the on Engineering in Medicine and Biology Society, pp. 5270-5273, IEEE, August 2008.

[25] M. Di Rienzo, F. Rizzo, G. Parati, G. Brambilla, M. Ferratini, and P. Castiglioni, "MagIC system: a new textile-based wearable device for biological signal monitoring applicability in daily life and clinical setting," in Proceedings of the IEEE 27th Annual International Conference of the Engineering in Medicine and Biology Society (EMBS '05), pp. 7167-7169, Shanghai, China, September 2005.

[26] V. Shnayder, B. R. Chen, K. Lorincz, T. R. F. Jones, and M. Welsh, "Sensor networks for medical care," in Proceedings of the 3rd International Conference on Embedded Networked Sensor Systems (SenSys '05), vol. 5, p. 314, San Diego, Calif, USA, November 2005.

[27] E. Montón, J. F. Hernandez, J. M. Blasco et al., "Body area network for wireless patient monitoring," IET Communications, vol. 2, no. 2, pp. 215-222, 2008.

[28] B. Gyselinckx, J. Penders, and R. Vullers, "Potential and challenges of body area networks for cardiac monitoring," Journal of Electrocardiology, vol. 40, no. 6, supplement 1, pp. S165-S168, 2007.

[29] N. Oliver and F. Flores-Mangas, "HealthGear: a real-time wearable system for monitoring and analyzing physiological signals," in Proceedings of the International Workshop on Wearable and Implantable Body Sensor Networks (BSN '06), pp. 61-64, Cambridge, Mass, USA, April 2006.

[30] P. Leijdekkers and V. Gay, "A self-test to detect a heart attack using a mobile phone and wearable sensors," in Proceedings of the 21st IEEE International Symposium on Computer-Based Medical Systems (CBMS '08), pp. 93-98, IEEE, Jyvaskyla, Finland, June 2008.

[31] K. Wac, R. Bults, B. Van Beijnum et al., "Mobile patient monitoring: the MobiHealth system," in Proceedings of the Annual International Conference Engineering in Medicine and Biology Society, pp. 1238-1241, Minneapolis, Minn, USA, September 2009.

[32] S. Jiang, Y. Cao, S. Iyengar et al., "CareNet: an integrated wireless sensor networking environment for remote healthcare," in Proceedings of the ICST 3rd International Conference on Body Area Networks (BodyNets '08), article 9, Tempe, Ariz, USA, 2008.

[33] T. Sheltami, A. Mahmoud, and M. Abu-Amara, "Warning and monitoring medical system using sensor networks," in Proceedings of the Saudi 18th National Computer Conference (NCC '06), pp. 63-68, Riyadh, Saudi Arabia, March 2006.

[34] E. Farella, A. Pieracci, L. Benini, L. Rocchi, and A. Acquaviva, "Interfacing human and computer with wireless body area sensor networks: the WiMoCA solution," Multimedia Tools and Applications, vol. 38, no. 3, pp. 337-363, 2008.

[35] J. Nehmer, M. Becker, A. Karshmer, and R. Lamm, "Living assistance systems-an ambient intelligence approach," in Proceedings of the 28th International Conference on Software Engineering (ICSE '06), pp. 43-50, Shanghai, China, May 2006.

[36] I. Ramachandran, A. K. Das, and S. Roy, "Analysis of the contention access period of IEEE 802.15.4 MAC," ACM Transactions on Sensor Networks, vol. 3, no. 1, article 4, 2007.

[37] G. Anastasi, M. Conti, and M. Di Francesco, "A comprehensive analysis of the MAC unreliability problem in IEEE 802.15.4 
wireless sensor networks," IEEE Transactions on Industrial Informatics, vol. 7, no. 1, pp. 52-65, 2011.

[38] G. Anastasi, M. Conti, and M. Di Francesco, "The MAC unreliability problem in IEEE 802.15.4 wireless sensor networks," in Proceedings of the 12th ACM International Conference on Modeling, Analysis, and Simulation of Wireless and Mobile Systems (MSWiM '09), pp. 196-203, Tenerife, Spain, October 2009.

[39] J.-G. Ko, Y.-H. Cho, and H. Kim, "Performance evaluation of IEEE 802.15.4 MAC with different backoff ranges in wireless sensor networks," in Proceedings of the 10th IEEE Singapore International Conference on Communications Systems (ICCS '06), pp. 1-5, Singapore, November 2006.

[40] T. H. Kim and S. Choi, "Priority-based delay mitigation for event-monitoring IEEE 802.15.4 LR-WPANs," IEEE Communications Letters, vol. 10, no. 3, pp. 213-215, 2006.

[41] M. Kim, C. H. Kang, and M. Kim, "Priority-based servicedifferentiation scheme for IEEE 802.15. 4 sensor networks in non-saturation environments," IEEE Transactions on Vehicular Technology, vol. 59, no. 7, pp. 3524-3535, 2010.

[42] J. Bhar, "A Mac protocol implementation for wireless sensor network," Journal of Computer Networks and Communications, vol. 2015, Article ID 697153, 12 pages, 2015.

[43] A. Koubaa, M. Alves, B. Nefzi, and Y. Q. Song, "Improving the IEEE 802.15.4 slotted CSMA/CA MAC for time-critical events in wireless sensor networks," Tech. Rep., IEEE, 2006.

[44] J. G. Ko, J. G. Cho, and H. Kim, "Performance evaluation of IEEE 802.15.4 MAC with different backoff ranges in wireless sensor networks," in Proceedings of the 10th IEEE Singapore International Conference on Communication Systems (ICCS '06), pp. 1-5, Singapore, October 2006.

[45] A. Koubaa, M. Alves, and E. Tovar, "A comprehensive simulation study of slotted CSMA/CA for IEEE 802.15.4 wireless sensor networks," in Proceedings of the IEEE International Workshop on Factory Communication Systems, pp. 183-192, IEEE, June 2006.

[46] S. Kutty and J. A. Laxminarayan, "Towards energy efficient protocols for wireless body area networks," in Proceedings of the 5th International Conference on Industrial and Information Systems (ICIIS '10), pp. 31-34, IEEE, Mangalore, India, August 2010.

[47] K. Hur, W.-S. Sohn, J.-K. Kim, and Y. Lee, "Novel MAC protocol and middleware designs for wearable sensor-based systems for health monitoring," International Journal of Distributed Sensor Networks, vol. 2013, Article ID 404168, 15 pages, 2013.

[48] N. Mouzehkesh, T. Zia, S. Shafigh, and L. Zheng, "D²MAC: dynamic delayed medium access control (MAC) protocol with fuzzy technique for wireless body area networks," in Proceedings of the IEEE International Conference on Body Sensor Networks (BSN '13), pp. 1-6, Cambridge, Mass, USA, May 2013.

[49] J. Yuan, C. Li, and W. Zhu, "Energy-efficient MAC in wireless body area networks," in Proceedings of the International Conference on Information Science and Technology Applications (ICISTA '13), June 2013.

[50] F. Xia, L. Wang, D. Zhang, X. Zhang, and R. Gao, "Ada-MAC: an adaptive MAC protocol for real-time and reliable health monitoring," in Proceedings of the IEEE International Conference on Cyber Technology in Automation, Control, and Intelligent Systems (CYBER '12), pp. 203-208, Bangkok, Thailand, May 2012.
[51] R. H. Kim and J. G. Kim, "Delay reduced MAC protocol for bio signal monitoring in the WBSN environment," in Advanced Science and Technology Letters, pp. 42-46, 2015.

[52] J. S. Yoon, G.-S. Ahn, S.-S. Joo, and M. J. Lee, "PNP-MAC: Preemptive slot allocation and non-preemptive transmission for providing QoS in body area networks," in Proceedings of the 7th IEEE Consumer Communications and Networking Conference (CCNC '10), pp. 1-5, IEEE, Las Vegas, Nev, USA, January 2010. 


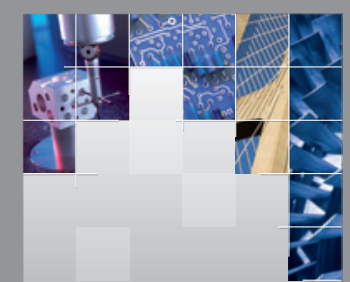

\section{Enfincering}
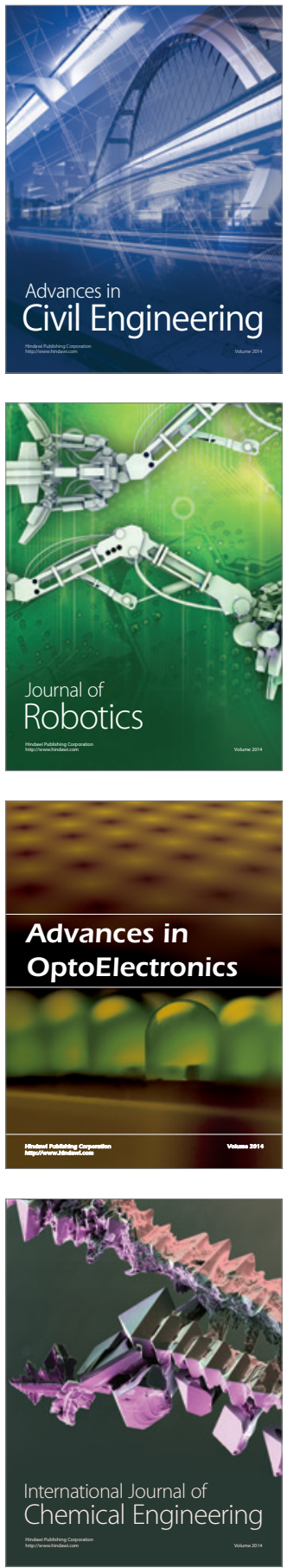

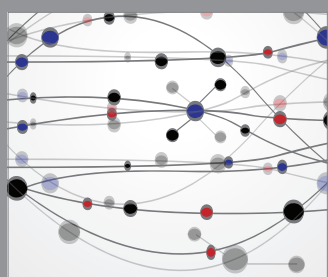

The Scientific World Journal

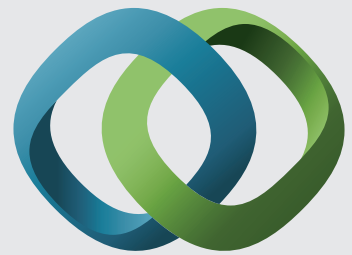

\section{Hindawi}

Submit your manuscripts at

http://www.hindawi.com
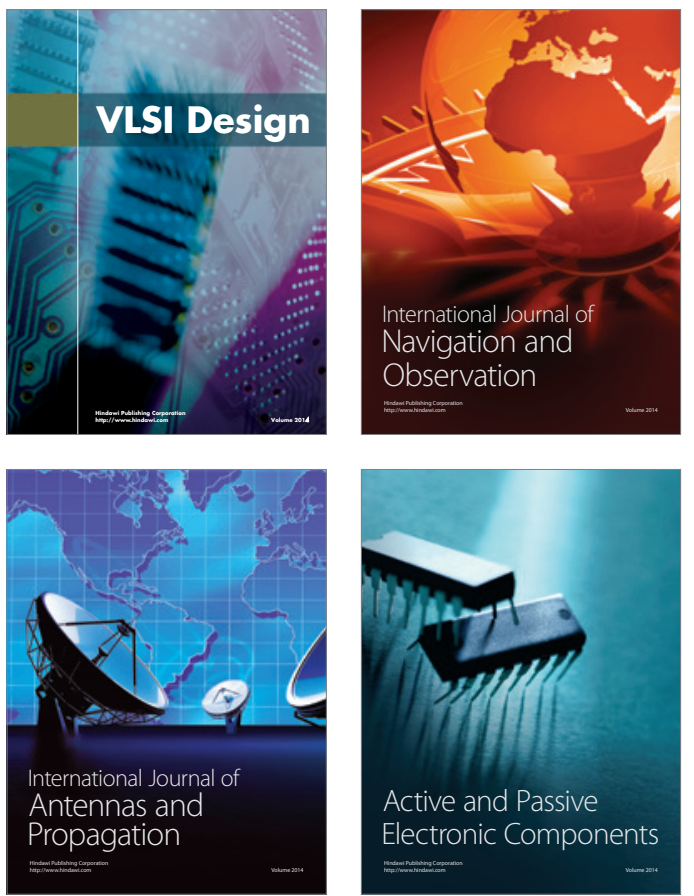
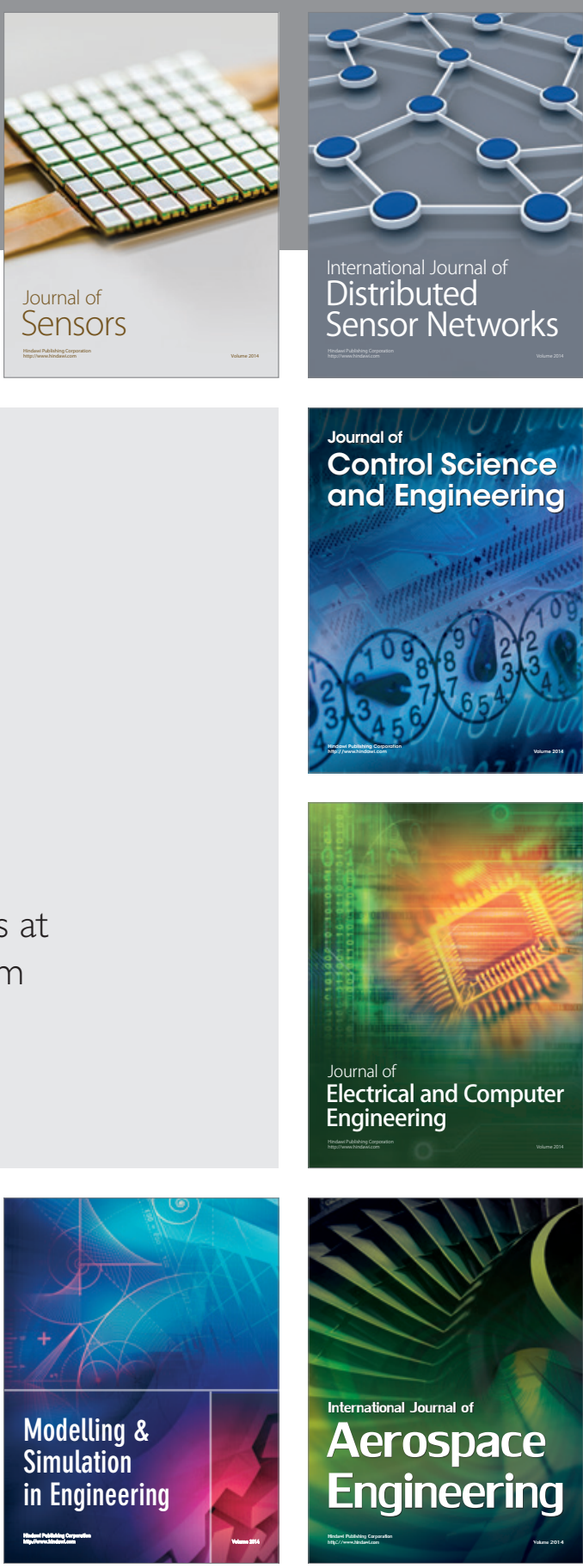

International Journal of

Distributed

Sensor Networks

Journal of

Control Science

and Engineering
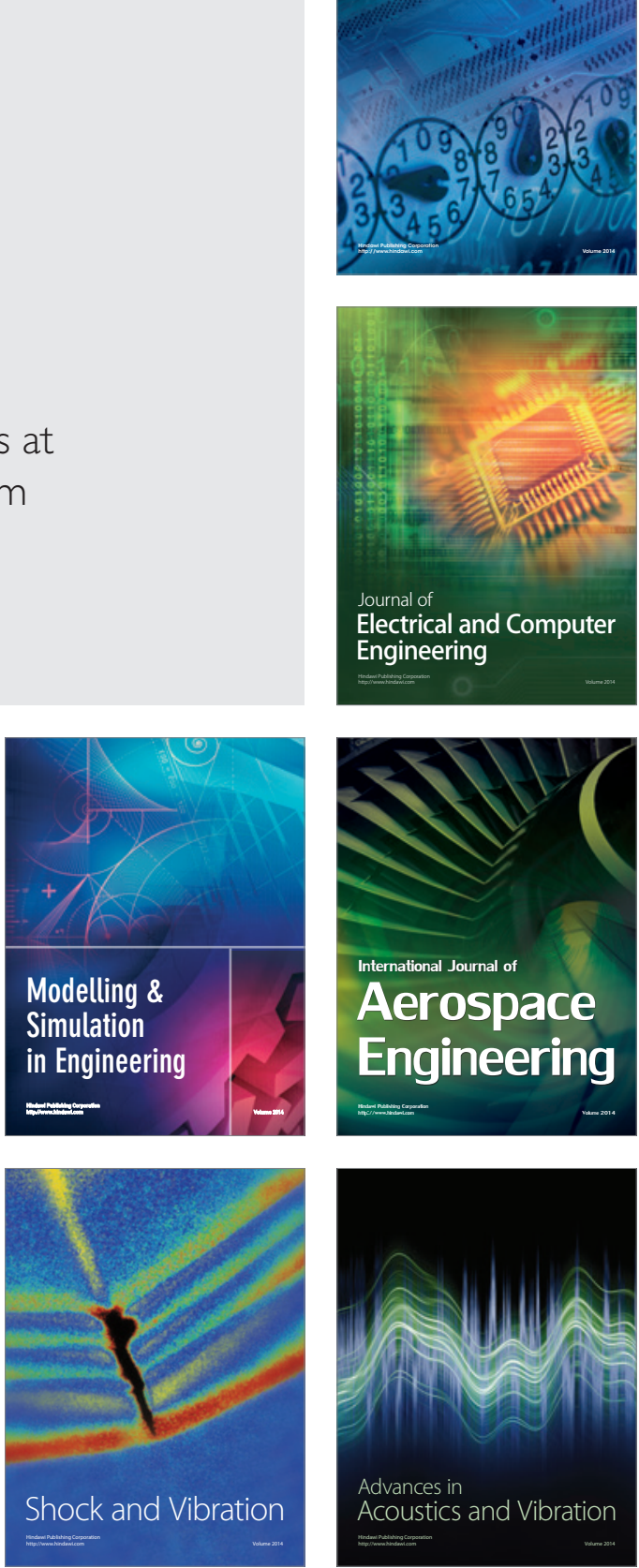